PALEO

Revue d'archéologie préhistorique

$18 \mid 2006$

Varia

\title{
Nouvelle lecture géologique du site paléolithique du Pech-de-l'Azé II (Dordogne, France)
}

New geological reading of the Paleolithic site of Pech-de-l'Azé II (Dordogne, France)

Jean-Pierre Texier

\section{OpenEdition}

Journals

Édition électronique

URL : http://journals.openedition.org/paleo/339

DOI : 10.4000/paleo.339

ISSN : 2101-0420

Éditeur

SAMRA

Édition imprimée

Date de publication : 1 décembre 2006

Pagination : 217-236

ISSN : 1145-3370

Référence électronique

Jean-Pierre Texier, « Nouvelle lecture géologique du site paléolithique du Pech-de-l'Azé II (Dordogne, France) », PALEO [En ligne], 18 | 2006, mis en ligne le 23 avril 2009, consulté le 07 juillet 2020. URL : http://journals.openedition.org/paleo/339; DOI : https://doi.org/10.4000/paleo.339

\section{(c) (1) (9)}

PALEO est mis à disposition selon les termes de la licence Creative Commons Attribution - Pas d'Utilisation Commerciale - Pas de Modification 4.0 International. 


\title{
NOUVELLE LECTURE GÉOLOGIQUE DU SITE PALÉOLITHIQUE DU PECH-DE-L'AZÉ II (Dordogne, France)
}

\author{
Jean-Pierre TEXIER ${ }^{(1)}$
}

\begin{abstract}
Résumé : Cette nouvelle approche géologique du site du Pech-de-l'Azé II a permis de mettre en évidence sept phases évolutives principales. La première date d'une période indéterminée de la fin du Tertiaire ou du début du Quatemaire. Au cours de la deuxième, se mettent en place des sables fluviatiles endokarstiques ; leur âge est également incertain. La troisième phase, dominée par le nuissellement associé à une éboulisation modée et un gélisol peu profond, a été rapportée à la première partie du stade isotopique de l'oxygène 6 (OIS 6). Lors de la quatrième, en liaison avec une intensification du froid, l'éboulisation devient prédominante et un gélisol profond se développe dans les dépôts sous-jacents ; cet épisode a été attribué à la partie supérieure de l'OIS 6 . Ensuite, lors de la phase 5 corrélée avec l'OIS 5, le ruissellement devient à nouveau prépondérant. Enfin, les phases 6 et 7 correspondent, pour l'essentiel, à une évolution diagénétique des dépôts antérieurs avec formation d'un gélisol profond (OSI 4 ou/et 2) puis, développement d'une importante bioturbation et de phénomènes de carbonatation (OIS 1).

Le ruissellement, potentiellement très perturbateur vis-à-vis des documents archéologiques, joue un rôle majeur dans la mise en place des unités sédimentaires 2 et 4 qui contiennent les niveaux acheuléens (niveaux 6 à 9) et moustériens (niveaux 2 à 4). Ceci explique probablement en partie l'incohérence apparente des dates ESR obtenues. Ce constat incite également à être relativement circonspect vis-à-vis de l'intégrité et de la pertinence des niveaux archéologiques définis dans ce site.
\end{abstract}

Mots-clés : Processus de formation des sites, Paléolithique inférieur et moyen, Pech-de-l'Azé II.

Key-words : Site formation processes, Middle and Lower Palaeolithic, Pech-de-l'Azé II.

\section{Abridged english version \\ NEW GEOLOGICAL READING OF THE PALAEOLITHIC SITE OF PECH-DE-L'AZÉ II (Dordogne, France)}

Discovered in the 1950s (Bordes et Bourgon 1951; Bordes 1954-1955), Pech-de-l'Azé II is a key prehistoric site in the Périgord in which the «Acheuléen méridional» is defined (Bordes 1971), as well as the different theories aiming to explain the variability of the Middle Palaeolithic (Bordes 1953 et 1981; Binford and Binford 1966 et 1969; Rolland 1981; Dibble 1987; Dibble and Mellars 1992; Mellars 1996).Micromorphological and chronological approaches carried out at the end of the 1970s (Goldberg 1979) and at the beginning of the 1990s (Grün and Stringer 1991; Grün et al. 1991) (fig. 1) have highlighted contradictions with the interpretations proposed by Bordes (1954-55), then by Laville (1973) (table 1). This led me to undertake new geological works in using the recent improvements made in the characterization and identification of continental sedimentary processes.

\section{METHODS}

This work is mainly based on descriptive methods, i.e. analysis of geomorphologic context and stratigraphic studies. They were completed by granulometric and micromorphological analysis. Fabric analysis were not performed because most of the rock fragments that crop out on sections, have an elongation index (Johanson 1963) lower than 1.7.

\section{GEOMORPHOLOGIC CONTEXT}

The site of Pech-de-l'Azé II is located $5 \mathrm{~km}$ southwest from the small town of Sarlat, in the commune of Carsac (Dordogne) (fig. 2). It occupies the NW entrance of a karstic passage (fig. 3) carved into a coniacian calcareous hill which is capped with a clayey sandy weathering mantle. It lies at about $140 \mathrm{~m}$ asl, 25 to $30 \mathrm{~m}$ above the bottom of a small dry valley (the Fage Valley), which joins that of the Enea river, a tributary of the Dordogne River.

\section{LITHOSTRATIGRAPHY}

We observe from the bottom to the top (fig. 4, 5 and 6):

Unit 1 (= level 10 of Laville):

Visible over a thickness of 10 to $30 \mathrm{~cm}$. Graded cross-bedded quartzose sands containing locally siliceous granules and/or granules of ferruginous sandstone. Colour : reddish yellow (7.5 YR 6/8 to 7/8). Some beds are cemented by carbonates. 
Unit 2 (= Levels 9 to 6 of Laville)

Thickness: 35 to $80 \mathrm{~cm}$. Diamicton with a matrix-supported texture or, more locally, with a clast-supported texture. Rock-fragments are heterometric, rounded and have no preferred orientation. They comprise limestone and some speleothem fragments. The matrix is a clayey sand. Its colour is variable: strong brown (7.5 YR 5/6 to 5/8), yellowish red (5 YR 5/6 to 4/6), reddish yellow (7.5 YR 6/6). Locally, we can observe graded-bedded sandy levels, beds composed of phosphate granules and zones impregnated with carbonates. Occurrence of a well-developed platy structure. The lower limit is regular and clear to gradual.

Unit 3 (= Levels 5 and $X$ of Laville)

Thickness: $35 \mathrm{~cm}$ to $1 \mathrm{~m}$. Composed of angular calcareous spalls and slabs. The structure is openwork to partially openwork. More or less clayey quartzose sands partially infill the packing voids. Calcitic pendents are seen on the lower part of the rock-debris. A well-expressed platy structure develops in the matrix the colour of which is variable: yellow (10 YR 7/6 to 7/8), reddish yellow (7.5 YR 6/6) or yellowish red (5 YR 5/6). Involutions are locally visible. The lower limit is clear and weakly undulated.

Unit 4 ( = Levels 4 to 2 of Laville)

Thickness: 1,80 m. Bedded sands passing laterally or vertically to massive sands (fig. 6). The colour is variable: reddish yellow (5 YR 5/6 to 4/6), yellowish red (5 YR 5/6 to 4/6), yellow (10 YR 7/8), strong brown (7.5 YR 5/6 to 5/8) or, more rarely, grey and dark red $(2.5$ YR $3 / 6)$. Some beds are composed of phosphate granules. In the middle of the unit, occurrence of an incipient flowstone. A well-expressed platy structure is visible in the lower part of the unit. Bioturbation is important in the upper part of the deposits. Occurrence of various carbonated features: matrix impregnations, nodules, rhizoliths, pseudomyceliums.

\section{MORPHO-DYNAMIC AND DIAGENETIC}

\section{INTERPRETATION}

On the basis of field studies and analytic results, it is possible to describe the main evolutionary phases of the site (table 2).

The first phase comesponds to the formation of the cave. Occurrence of ceiling pockets lead to think that this episode occurs in a phreatic context (Bretz 1942; White 1988).

The second phase is characterised by the deposition of endokarstic fluvial sands (Unit 1). The fluvial origin of these deposits is inferred from their cross-bedded organisation and their good sorting (fig. 8).

The petrographic composition of these alluvia (quartz, ferruginous sandstone, siliceous gravels) as well as the etched appearance of numerous quartz grains (fig. 11) indicate that these sediments mainly come from the weathering mantle which capes the cretaceous hill. The ferruginous clayey coatings observed at microscopic scale (fig.11) are probably $p$ ro parte inherited from this formation.

Microsparite that locally cements unit 1 (fig. 11) precipitated in a vadose context. It corresponds to a late diagenetic process because it is not affected by the frozen soil which develops in the overlying deposits.

Speleothems found as fragments in units 2 and 3 probably formed during this evolutionary episode.

The third phase comesponds to the deposition of unit 3 during which the cave continues its evolution in a vadose context. From the general organisation of sediments, this unit results from runoff processes associated to moderate rockfalls.

Colour's variations of the matrix are probably linked to synsedimentary processes (difference in proportions of sediments coming from the weathering mantle and sediments originating from the granular disaggregation of the surrounding limestone, various amounts of organic matter) and diagenetic phenomena (carbonatations). As previously shown by Goldberg (1979), there is no feature characteristic of luvisol. The occurrence of speleothem fragments shows that unit 2 was emplaced inside the cave the entrance of which was situated westward of the present-day one.

The ovoïd structure observed at microscopic scale (fig. 12) reveals that a shallow frozen soil has probably accompanied the sedimentation (Bertran 1993). The platy structure, which is superimposed on ovoïds (fig. 12), is thought to be related to a further evolutionary phase (cf. Infra).

The forth evolutionary phase is heavily marked by cryogenic processes. Indeed, several data indicate that frost weathering played a major role in the accumulation of rock-fragments which compose unit 3: 1) The platy shape of rock-debris can be related to a freezing front penetrating parallel to the walls of the cave; 2 ) Generalization of rockfall process to the whole cave suggests the intervention of a climate-driven phenomenon; 3) The huge slabs contained in this unit have broken down into plates whose surface is parallel to the surface of the slabs.

Moreover, the platy structure observed in the matrix of units 3 and 2 (fig. 12 and 14) testifies to the development of a deep seasonal frozen soil, perhaps permafrost. Involutions noted in unit 3 can be ascribed to the same phenomenon and are interpretated as cryoturbation features.

The next phase (Phase 5) comesponds to the deposition of the upper unit (unit 4). This one displays lithofacies and sedimentary structure revealing that sedimentogenesis is again dominated by runoff allied to moderate rockfalls.

The incipient flowstone visible in the middle part of unit 4 (fig. 6) is related to dripping processes coming down from the cave ceiling. This phenomenon is also probably responsible for the erasing of bedded structures in this part of the site.

The following phase 6 is characterized by the development of the platy structure observed in the lower par part of the unit 4 (fig. 15). It can be associated to a deep seasonal frozen soil, perhaps permafrost. In the upper part of unit 4, these cryogenetic features were probably erased by the last diagenetic episode that affected the deposits (Phase 7, cf. infra). 
The last evolutionary phase (phase 7) is characterized by the collapse of the ceiling in the outer part of the cave, by important bioturbation of the upper part of deposits (fig. 16), and by the precipitation of carbonates which locally impregnate the matrix or form rhizoliths and pseudomyceliums.

\section{CHRONOLOGY OF THE DIFFERENT EVOLUTIONARY PHASES}

On the grounds of geological results and numerical datings carried out in this site (Schwarcz et Blackwell 1983; Grün et Stringer 1991;

Grün et al. 1991) (fig. 1), it is possible to propose the following chronological hypothesis (table 2) :

- The evolutionary phases 3 and 4 are contemporaneous with a cold, then a very cold, climatic environment. They probably represent the first and the last part of the OIS 6 respectively.

- Phase 5 is dominated by runoff processes and an incipient flowstone forms locally. No evidence of a cold climate was found. It has been attributed to the OIS 5 .

- Phase 6 is characterized by the development of a deep seasonal frozen soil, perhaps permafrost. It has been ascribed to the OIS 4 or/and to the OIS 2.

- The last phase, typified by important bioturbations and carbonatations, has been dated to OIS 1 .

There is no chronological marker to precisely situate the two first evolutionary phases in the Quatemary system.

\section{CONCLUSIONS}

This new geological approach has allowed the definition of seven evolutionary phases. The first one corresponds to the formation of the karst system; it dates to an unknown period of the Quatemary. Endokarstic fluvial sands sediment during the second phase the age of which is uncertain. Runoff processes and a shallow frozen soil characterize the third phase which has been ascribed to the first part of the OIS 6. During the forth phase, sedimentogenesis is dominated by rockfall processes and a deep seasonal frozen soil (or a permafrost) develops in the underlying deposits. This episode that appears to be colder than the former, has been attributed to the upper part of OIS 6. Then, runoff becomes predominant again; this fifth phase which displays no evidence of cold conditions, is probably contemporaneous with the OIS 5 . At least, the phases 6 and 7 are mainly diagenetic: first, a deep seasonal frozen soil, or permafrost, forms during the OIS 4 and/or the OIS 2, then bioturbation and carbonation processes occur mainly in the upper part of the stratigraphic series (OIS 1).

Runoff, which is potentially very damaging for archaeological documents (Lenoble 2005), plays a major role in the deposition of sedimentary units 2 and 4 which contain Acheulean levels (Levels 6 to 9 ) and Mousterian levels (levels 2 to 4). This noting incites to be cautious regarding the integrity and the relevance of archaeological levels formerly defined in this site.

\section{1 - INTRODUCTION}

Découvert en 1949 par F. Bordes et M. Bourgon (Bordes et Bourgon 1951 ; Bordes 1954-1955), le Pech-de-l'Azé II est un gisement clé pour la compréhension du Paléolithique ancien et moyen. Avec le site de CombeGrenal, il a servi de support à $F$. Bordes pour définir l'Acheuléen méridional (Bordes 1971). II a également été intégré à la plupart des synthèses qui ont été tentées pour expliquer la variabilité du Paléolithique moyen (Bordes 1953, 1981 ; Binford and Binford 1966, 1969 ; Rolland 1981 ; Dibble 1987 ; Dibble and Mellars 1992 ; Mellars 1996).

Néanmoins, les approches micromorphologiques (Goldberg 1979) puis chronologiques (Grün and Stringer 1991 ; Grün et al. 1991) menées dans ce site à la fin des années 70 et au début des années 90 , ont fait apparaître d'importantes contradictions avec les interprétations proposées par F. Bordes (1954-1955), puis par H. Laville (1973) (tabl. 1). Ainsi, l'existence des «paléosols» censés représenter les «interstadiaires rissiens» est infimǿe par la micromorphologie (cf. infra). En outre, alors que la chronostratigraphie

\begin{tabular}{|c|c|c|}
\hline Auteurs & $\begin{array}{l}\text { Nb. d'unités } \\
\text { strati. } \\
\text { identifiées }\end{array}$ & Interprétations \\
\hline $\begin{array}{l}\text { - Bordes F (1954) } \\
\text { - Bordes F. et Prat F. } \\
\text { (1965) }\end{array}$ & 10 couches & $\begin{array}{ll}\text { - } & \text { Couches } 1 \text { à } 5: \text { Würm I } \\
\text { - } & \text { "Sol" sur les couches } 6 \text { et } 7: \text { Riss-Würm } \\
\text { - } & \text { Couches } 6 \text { à } 9: \text { Riss } \\
\text { - } & \text { Des "sols" caractérisent les } \\
& \text { Insterstadiaires rissiens }\end{array}$ \\
\hline - Laville H. (1973) & $\begin{array}{l}20 \text { niveaux } \\
\text { (Subdivisions } \\
\text { des couches } \\
\text { définies par } F \text {. } \\
\text { Bordes) }\end{array}$ & $\begin{array}{ll}\text { - } & \text { Couches } 2 \text { à } 5: \text { Würm I subdivisé en } 9 \\
& \text { phases climatiques } \\
\text { - } & \text { Couche X : Riss III } \\
\text { - } & \text { Couches } 6 \text { et } 7: \text { Riss II subdivisé en } 4 \\
& \text { phases climatiques } \\
\text { - } & \text { Couches } 8 \text { et } 9: \text { Riss I subdivisé en } 2 \\
\text { phases climatiques } \\
\text { - Couche } 10: \text { Mindel-Riss } \\
\text { - } \quad \text { Des "sols" caractérisent les interstades } \\
\text { rissiens et l'interglaciaire Riss-Würm }\end{array}$ \\
\hline
\end{tabular}

Tableau 1 - Nombre d'unités stratigraphiques et interprétations des dépôts du Pech-de-l'Azé Il selon Bordes (1954), Bordes et Prat (1965) et selon Laville (1973).

Table 1- Number of stratigraphic units and interpretation of Pech-de-l'Azé II deposits following Bordes (1954), Bordes and Prat (1965) and Laville (1973). 


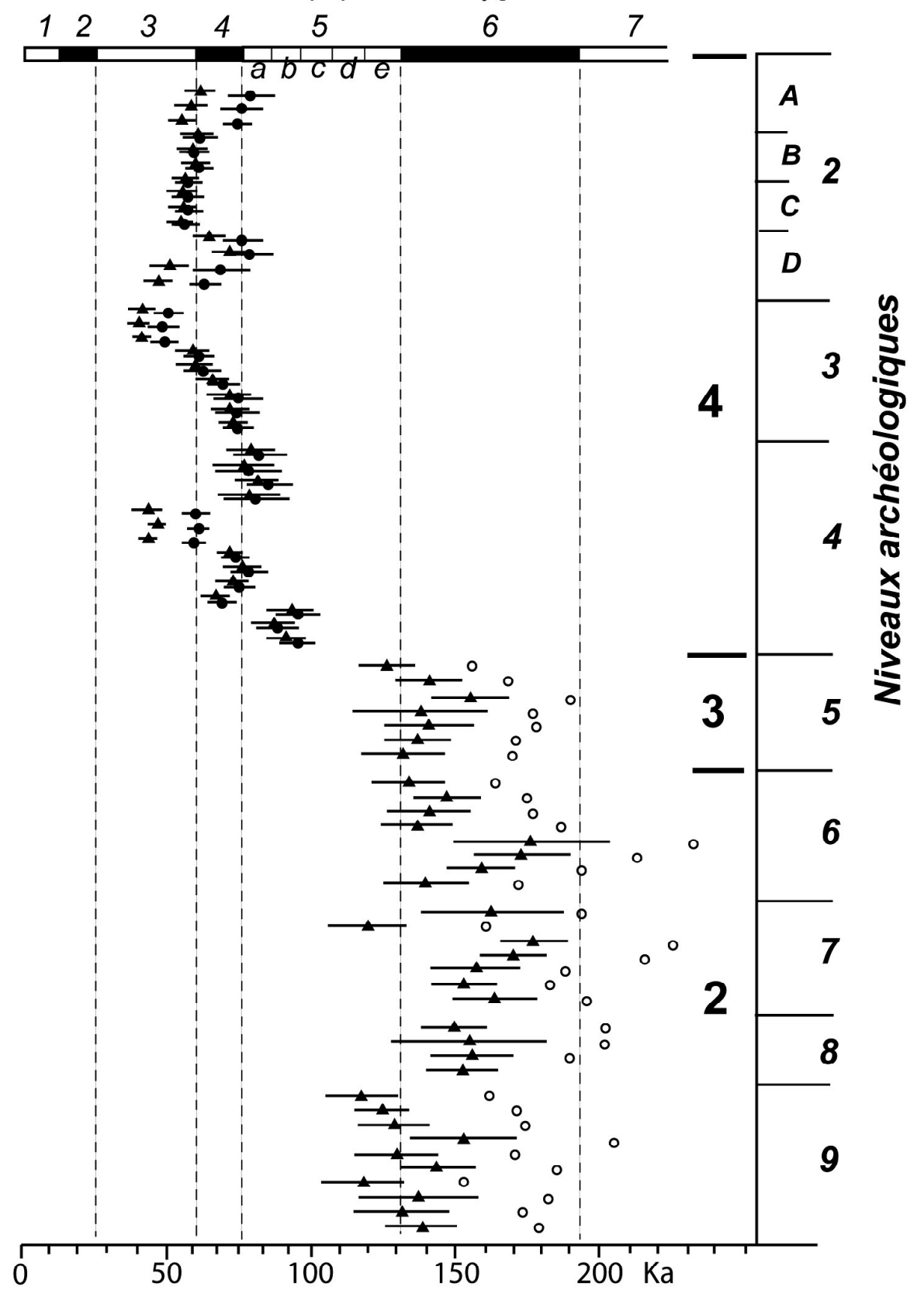

Figure 1 - Datations ESR obtenues dans le site de Pech-del'Azé II (D'après Grün et Stringer 1991, modifié). Triangles pleins : dates EU («Early Uptake») avec barres d'erreurs

Cercles pleins : dates $L U$ ("Linear Uptake») avec barres d'erreurs

Cercles évidés : dates $L U$ estimées.

Les chiffres en gras 2, 3, 4, placés en vis-à-vis de ceux des niveaux archéologiques correspondent aux unités lithostratigraphiques définies dans le texte.

Figure 1 - ESR age results from Pech-de-l'Azé II (From Grün and Stringer, 1991, modified)

Triangles: EU ages with errors. Closed circles: $L U$ ages with errors

Open circles : LU age estimates.

Bold numbers 2, 3, 4, facing those of archaeological levels correspond to lithostratigraphic units defined in the text.

Ce constat m'a amené à reprendre l'étude géologique de ce site-clé sur de nouvelles bases et en intégrant les progrès récents réalisés dans la connaissance et l'identification des processus sédimentaires continentaux. Ces travaux ont été réalisés dans le cadre d'un programme collectif de recherche (PCR) menés en collaboration avec $F$. Delpech et J.-Ph. Rigaud, et intitulé «Litho- et bio-stratigraphie de quelques sites de référence périgourdins». Les résultats obtenus ont été exposés dans le rapport final (Texier et al. 1999) ou présentés à l'occasion de colloques et d'excursions (Texier 2001a, 2001b et 2003a Texier et al. 2004). Cependant, ils n'ont jamais fait l'objet d'une publication exhaustive. C'est ce que je me propose de faire pour le Pech-de-l'Azé II dans le cadre de cet article. Les principaux buts visés par cette étude sont d'établir la lithostratigraphie du site, de caractériser les processus géologiques responsables de sa genèse et d'identifier les principales phases morphosédimentaires de son évolution. Une tentative de «calage» chronologique de ces différents épisodes est également présentée.

\section{2 - LES TRAVAUX GÉOLOGIQUES ET CHRONOLOGIQUES ANTÉRIEURS}

Les premiers travaux géologiques ont été réalisés en 1951 par F. Bordes et M. Bourgon qui donnent une description stratigraphique sommaire du site.

En 1973, H. Laville propose une nouvelle lecture chronostratigraphique des dépôts : vingt niveaux correspondant 


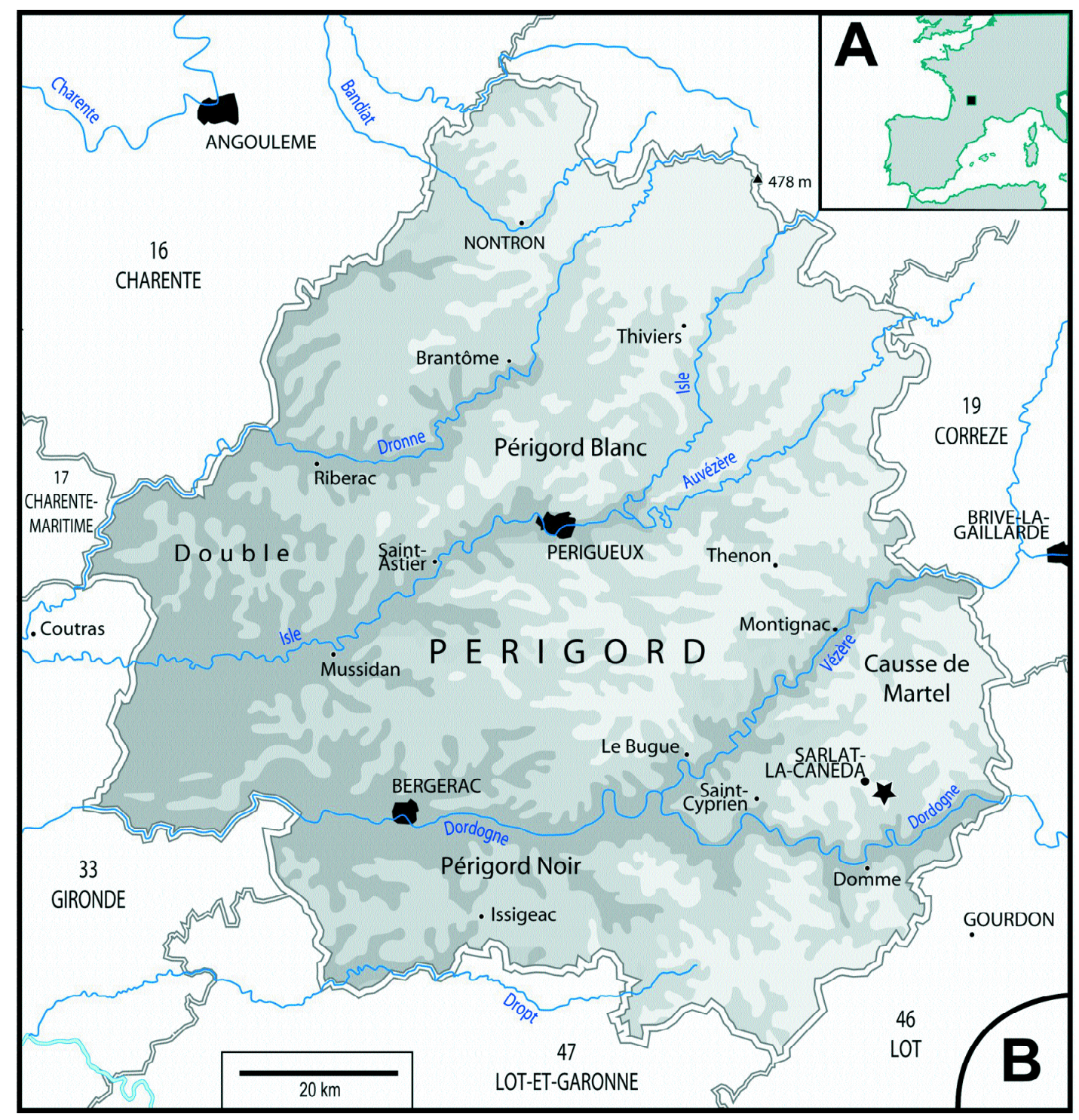

Figure 2 -

Localisation

géographique

du Pech-de-

l'Azé II (étoile).

Figure 2 -

Geographic

location

of Pech-de-

l'Azé Il (star).

pour l'essentiel à des subdivisions des couches identifiées par Bordes et Bourgon, sont individualisés.

Les principales interprétations proposées par ces auteurs (Bordes 1954 ; Bordes et Prat 1965 ; Laville 1973) sont regroupées sur le tableau 1.

Par la suite, Goldberg (1979) effectue une étude micromorphologique des sédiments. Parmi les résultats obtenus, on soulignera notamment la mise en évidence d'organisations liées à un gel profond («banded fabric») et l'absence de traits pédologiques en place dans les niveaux interprétés par Laville (op.cit.) comme des paléosols.

En 1983, Schwarcz et Blackwell obtiennent un âge Th / U de $103+30 /-25$ Ka pour un plancher stalagmitique incipient inclus dans la couche 2 .

Environ une décennie plus tard, en 1991, quatre-vingts dates ESR concernant les couches 2 à 9 sont publiées (Grün et Stringer 1991; Grün et al. 1991). Ces dates forment deux groupes distincts (fig. 1) : un groupe de dates, concernant les couches 9 à 5 , est centré principalement sur le stade isotopique 6 mais «déborde» aussi sur la fin du stade 7 et le début du stade 5 ; un autre groupe de dates, portant sur les couches 4 à 2 , se répartit entre la fin du stade 5 et le début du stade 3. À l'intérieur de ces deux groupes, les dates sont indépendantes de la position stratigraphique des couches auxquelles elles se rapportent : ainsi, la couche 5 foumit des dates comparables à la couche 9 ; il en va de même pour les couches 4 et 2 (fig. 1).

\section{3 - MÉTHODES D'ÉTUDE}

L'approche méthodologique développée ici est classique et fait appel à des méthodes principalement descriptives.

Les dépôts étudiés ont été situés dans leur contexte géomorphologique à partir d'observations de terrain et de l'analyse de documents cartographiques : cartes topographiques IGN à $1 / 25000$ de Sarlat Ouest $\left(n^{\circ} 2036\right)$ et carte géologique à 1/80 000 de Bergerac. 
Les unités stratigraphiques ont été définies à partir de critères simples, directement appréciables sur le terrain : caractéristiques générales (épaisseur, pente, morphologie et type de contact), organisation générale des dépôts (massive, litée...), couleur et structure des sédiments (ouverte, semi-ouverte, à support clastique, à support matriciel), dimensions, nature et morphologie des fragments rocheux, description et distribution des traits diagénétiques (concrétions carbonatées, structure lamellaire...). Les couleurs ont été prises sur sédiment humide à l'aide du code Munsell. La granulométrie de la matrice (i.e. les particules inférieures à $2 \mathrm{~mm}$ ) a été établie par diffractométrie laser (appareil de type Malvern 2600) et par tamisage mécanique.

L'organisation microscopique des sédiments a été étudiée à partir de lames minces de grandes dimensions taillées dans des blocs orientés, imprégnés sous vide par une résine polyester selon la technique préconisée par Guilloré (1980). La terminologie utilisée pour la description de ces lames est adaptée de celle définie par Bullock et al. (1985).

Les analyses de fabrique n'ont pas pu être réalisées car les fragments rocheux affleurant sur les coupes sont principalement compacts ou aplatis et la majorité d'entre eux présente un taux d'allongement $a / b$ (Johansson 1963) inférieur à 1,7. Or, cette valeur représente une limite en deçà de laquelle les mesures d'orientation des objets ne sont plus significatives.

\section{4 - L'ENVIRONNEMENT GÉOMORPHOLOGIQUE}

Le site du Pech-de-l'Azé II est localisé à $5 \mathrm{~km}$ au sud-est de Sarlat, sur la commune de Carsac (Dordogne) (fig. 2). II est associé à l'une des ouvertures (celle orientée au NO) d'un couloir karstique (fig. 3) creusé dans une butte de calcaire coniacien (un «pech» selon la désignation locale), couronnée par des altérites sablo-argileuses rouges. Situé à environ $140 \mathrm{~m}$ d'altitude, il domine de 25 à $30 \mathrm{~m}$ le fond d'une petite vallée localement sèche (vallée de Farge) qui rejoint celle de l'Enéa, affluent de rive droite de la Dordogne.

\section{5 - LITHOSTRATIGRAPHIE}

L'établissement de la lithostratigraphie du site s'appuie sur l'étude des coupes $n^{\circ} 2,3$, 4 et 5 dont la localisation est précisée sur la figure 2 (numérotation donnée par $\mathrm{H}$. Laville 1973). Les coupes $n^{\circ} 1$ et 6 ne sont plus observables. Les coupes $n^{\circ} 2$ et 5 donnent une bonne idée des différents facies observés dans ce gisement ; elles ont donc été représentées sur les figures 4 et 5 . Quatre unités sédimentaires ont été identifiées. On observe de bas en haut:

Unité 1 (= couche 10 de Laville) :

Visible uniquement à la base des différentes coupes sur une épaisseur de 10 à $30 \mathrm{~cm}$. Sables quartzeux lités, fins

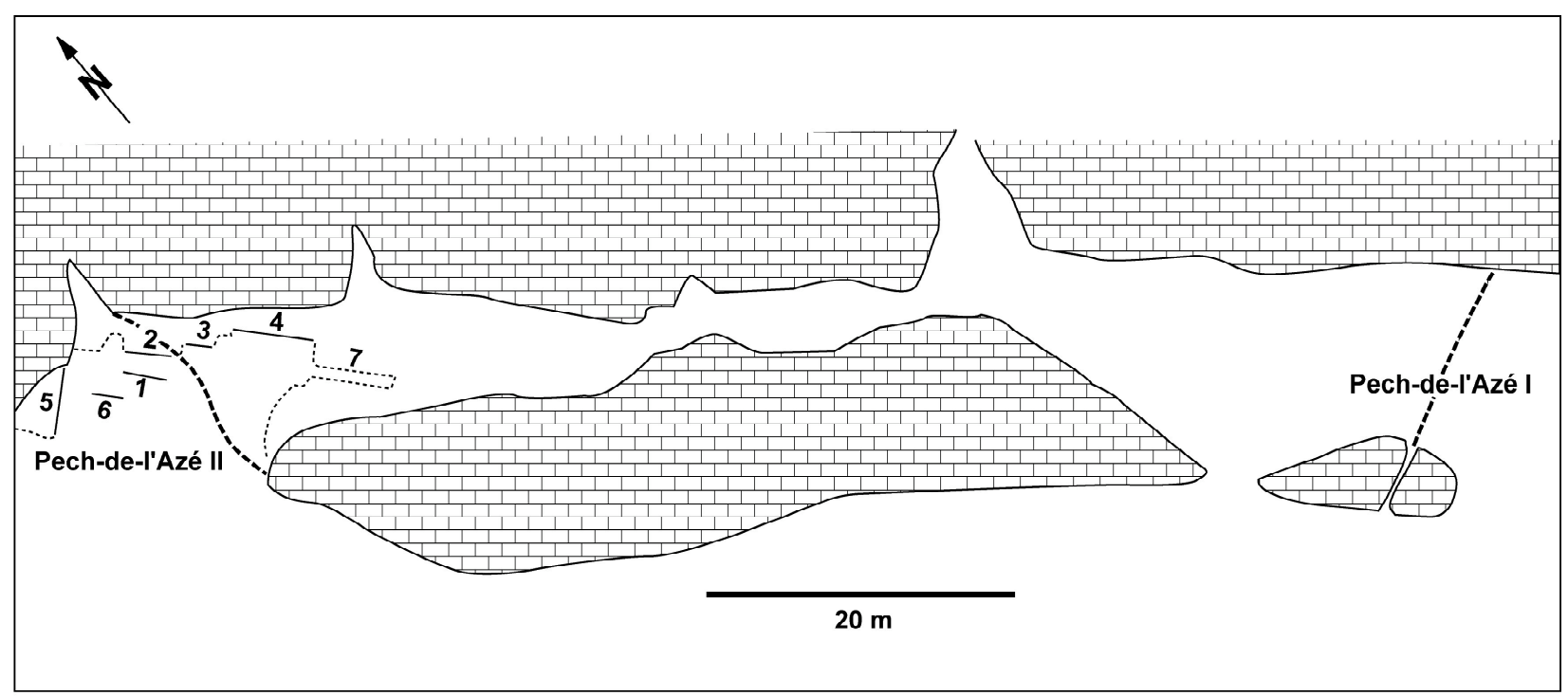

Figure 3 - Plan du conduit karstique et localisation des sites du Pech-de-l'Azé I et du Pech-de-l'Azé Il (d'après Laville, 1973, modifié). 1 à 6 : Numéro et situation des coupes étudiées par H. Laville (1973).

Figure 3 - Plan of the karstic passage and location of the sites of Pech-de-l'Azé I and Pech-de-l'Azé II (from Laville, 1973, modified). 1 to 6 : Number and situation of the sections studied by $H$. Laville (1973). 


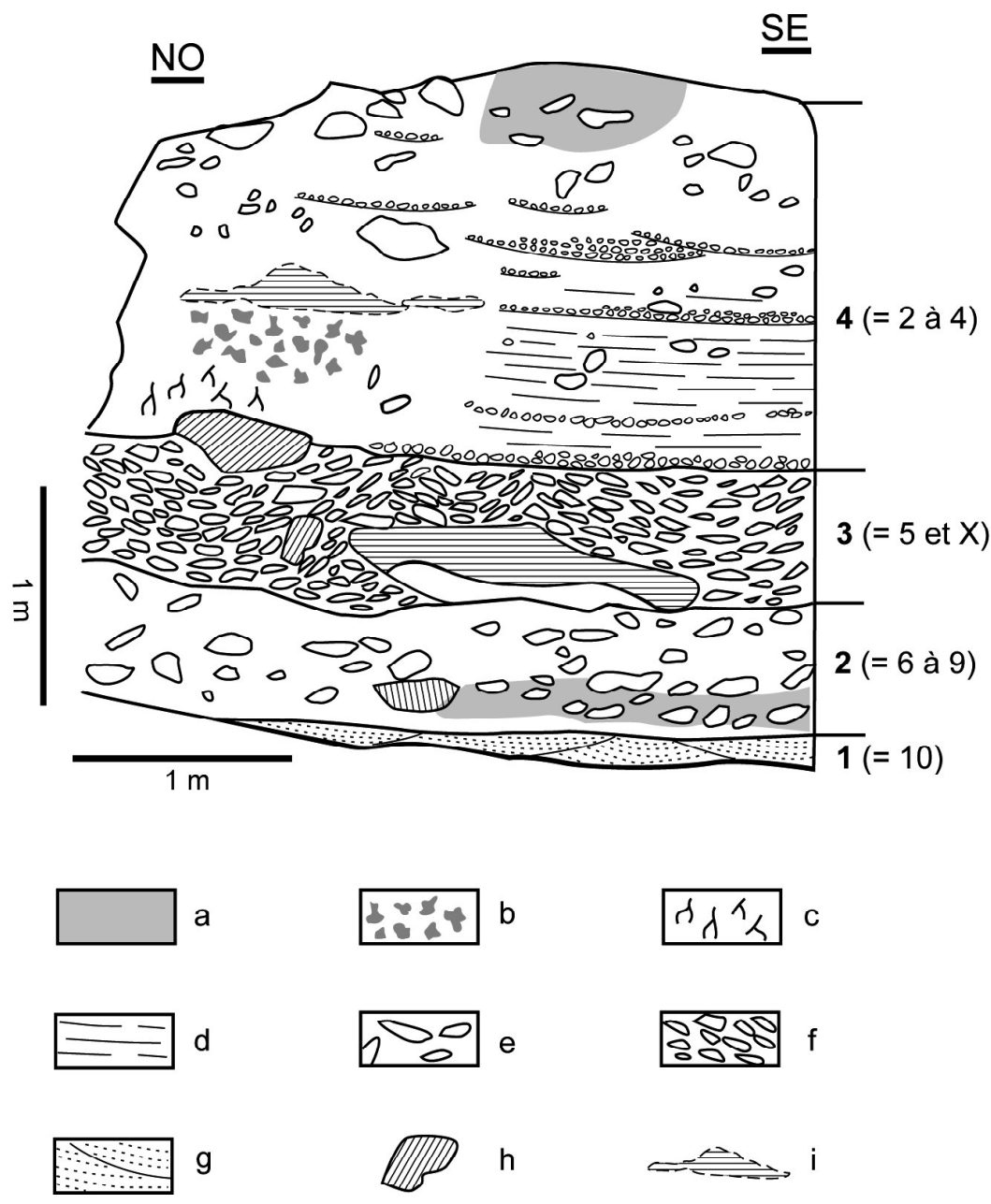

Figure 4 - Pech-de-l'Azé II.

Stratigraphie observée

sur la coupe $n^{\circ} 2$.

1, 2, 3, 4 : unités sédimentaires. Les chiffres entre parenthèses correspondent aux unités définies par H.Laville (1973)

a : imprégnations calcitiques -

$b$ : concrétions calcitiques -

$c$ : pseudomycelium

$d$ : dépôts lités -

e : diamicton -

$f$ : cailloutis semi-ouvert -

$g$ : sables à stratifications entrecroisées -

$h$ : fragments de spéléothèmes -

$i$ : spéléothème incipient.

Figure 4 - Pech-de-l'Azé II.

Stratigraphy of section 2. 1, 2, 3,

4: sedimentary units. Numbers in

brackets correspond to Laville's units.

a: calcitic impregnations -

b: calcitic concretions -

c: pseudomycelium

d: bedded deposits -

e: diamicton -

f: openwork rock-debris accumulation $g$ : cross-bededd sands -

$h$ : speleothem fragments -

i: incipient speleothem.

à grossiers, granoclassés, parfois associés à des granules siliceux altérés et à des granules de grès fernugineux. Stratification entrecroisée ou oblique. Couleur générale : jaune rouge (7.5 YR 6/8 à 7/8). Certains lits sont consolidés par des carbonates.

Unité 2 ( = couches 9 à 6 de Laville) :

Epaisseur : 35 à $80 \mathrm{~cm}$. Diamicton présentant une structur re à support matriciel ou, plus localement, à support clastique. Les éléments grossiers sont très hétérométriques ; leurs dimensions varient de $2-3 \mathrm{~cm}$ à $30-40 \mathrm{~cm}$. Ils ne sont pas orientés et sont plus ou moins fortement émoussés. Ils comprennent essentiellement des calcaires et, localement (coupe 2), des fragments de spéléothèmes. La matrice est un sable quartzeux, plus ou moins argileux, dont la couleur est variable : brun vif (7.5 YR 5/6 à 5/8), rouge jaune (5 YR $5 / 6$ à 4/6), jaune rouge (7.5 YR 6/6). Des zones imprégnées de carbonates (coupe 2), des lits sableux granoclassés et des lits de granules phosphatés blanchâtres (coupe 4) s'observent localement. Présence d'une structure lamellaire généralement bien exprimée. Limite inférieure : régulière, nette à diffuse.
Unité 3 (= couches 5 et $X$ de Laville)

Epaisseur : varie de $35 \mathrm{~cm}$ à l'extrémité SO de la coupe 5 , à $1 \mathrm{~m}$ vers l'intérieur de la grotte (coupe 4). Formée de plaquettes et de dalles calcaires. Structure généralement semi-ouverte à ouverte, localement colmatée par un sable quartzeux plus ou moins argileux. La dimension des plaquettes varie de $2-3 \mathrm{~cm}$ à plusieurs décimètres ; celle des dalles peut atteindre jusqu'à $3 \mathrm{~m}$. Celles-ci sont présentes principalement vers l'intérieur de la cavité (coupe 4) et sont plus ou moins délitées sur place. Ces éléments rocheux sont principalement anguleux. Des fragments de planchers stalagmitiques et de stalactites sont associés aux fragments calcaires dans la coupe 2. On note localement la présence de revêtements calcitiques bourgeonnants («pendants») à la face inférieure des plaquettes. La matrice est de couleur variable : jaune (10 YR 7/6 à 7/8), jaune rouge (7.5 YR 6/6 ou rouge jaune (5 YR 5/6). Elle présente une structure lamellaire bien développée. Vers l'extérieur de la cavité (coupes 5 et 2), cette unité présente des involutions plus ou moins marquées et s'organise en cellules de taille variable (30 à $80 \mathrm{~cm}$ de large). Sur la coupe 5 , au voisi- 


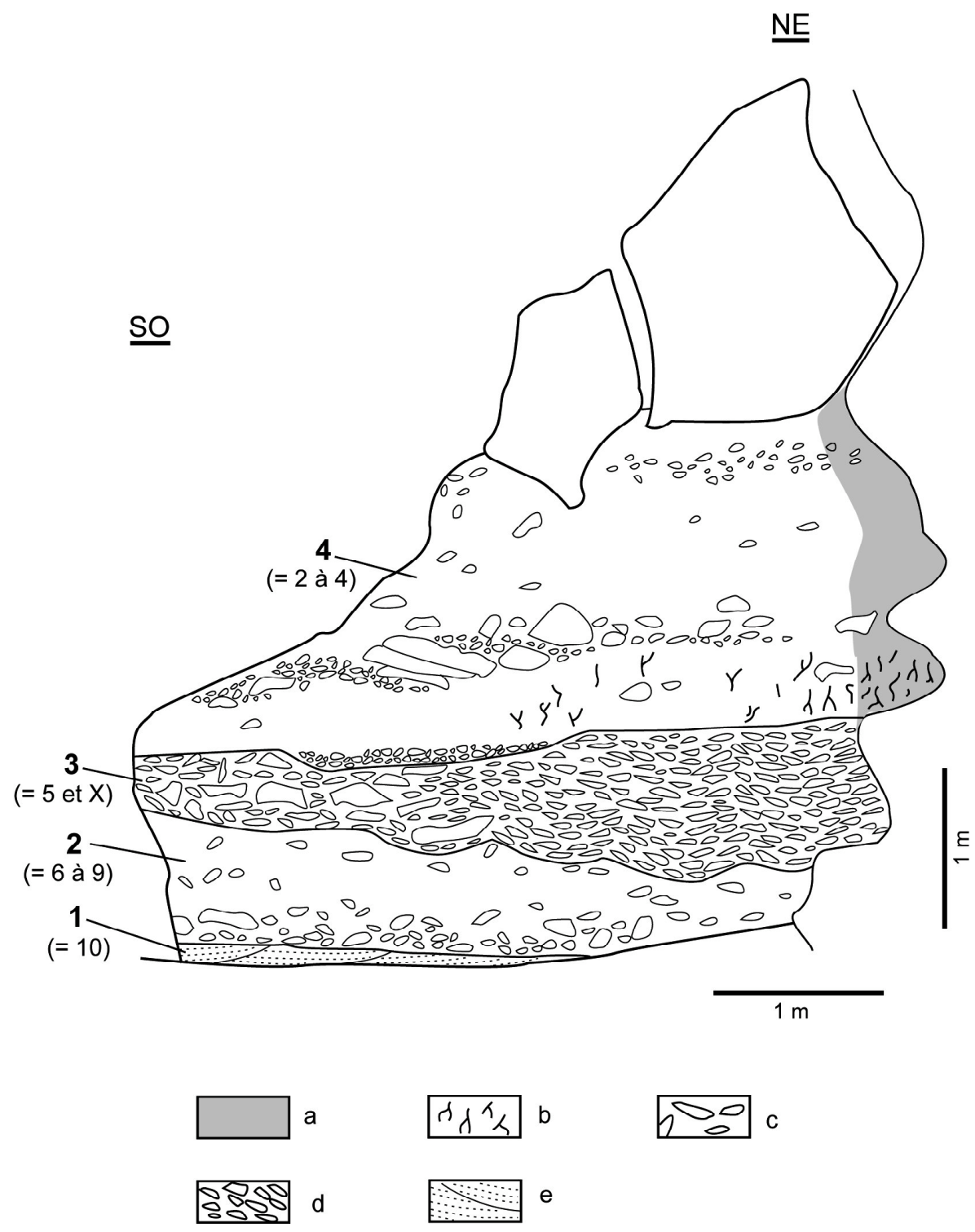

Figure 5 - Pech-de-l'Azé II. Stratigraphie observée sur la coupe $n^{\circ} 5$.

1, 2, 3, 4 : unités sédimentaires. Les chiffres entre parenthèses correspondent aux unités définies par $H$. Laville (1973).

a : imprégnation calcitique -

$b$ : pseudomycelium -

$c$ : diamicton

$d$ : cailloutis semi-ouvert e : sables à stratifications entrecroisées.

Figure 5 - Pech-de-l'Azé II. Stratigraphy of section 5 .

1, 2, 3, 4: sedimentary units. Numbers in brackets correspond to Laville's units.

a: calcitic impregnations -

b: pseudomycelium -

c: diamicton

d: openwork rock-debris accumulation -

e: cross-beded sands.

nage de la paroi calcaire, elle est affectée par une carbonatation assez prononcée. Limite inférieure : nette, faiblement ondulée.

Unité 4 ( = couches 4 à 2 de Laville)

Totalement fouillée dans la partie la plus interne de la cavité, elle ne s'observe plus que sur les coupes 2, 3 et 5 . Son épaisseur atteint 1,80 $\mathrm{m}$. Elle débute par un niveau discontinu de graviers et de petits cailloux calcaires arrondis, colmatés par des sables jaunes (10 YR 7/8) ; épais de 15 à $20 \mathrm{~cm}$, il remplit de petites dépressions au sommet de l'unité 3 et correspond au niveau 4D de Laville. Au-dessus, cette unité présente tantôt un faciès lité tantôt un faciès massif, avec des passages latéraux ou verticaux d'un type à l'autre.

Le faciès lité est bien représenté sur les coupes 2 et 3 (fig. 4 et 6 ). II est constitué de lits plans, d'épaisseur pluri-millimétriques à pluri-centimétriques et d'extension décimétrique à supramétrique. La plupart d'entre eux est formée de sables granoclassés, jaune rouge (5 YR 5/6 à 4/6), jaunes (10 YR 7/8) ou, plus rarement, gris par suite de la présence de matière organique et/ou de fragments charbonneux. On observe également quelques lits de granules phosphatés, blancs et friables.

Le faciès massif se rencontre sur toutes les coupes. II comprend des sables plus ou moins argileux associés à des quantités variables de fragments calcaires, émoussés et non orientés. Ceux-ci, dont la taille de $2-3 \mathrm{~cm}$ à $20-25$ $\mathrm{cm}$, sont généralement dispersés dans la masse mais peuvent, comme sur la coupe 5, former localement des lignes que l'on suit sur plusieurs mètres. Ces éléments rocheux ont tendance à devenir plus abondants vers le sommet de l'unité. La matrice présente des zones de couleurs différentes : rouge jaune (5 YR 5/6 à 4/6), brun vif (7.5 YR 5/6 à $5 / 8$ ) et, plus rarement, rouge foncé (2.5 YR 3/6). Le passage d'une zone colorée à une autre est graduel ; il peut se faire dans le sens vertical ou latéral. Dans la partie NO de 
la coupe 2, la zone médiane de cette unité contient une amorce de plancher stalagmitique qui surmonte une zone à nodules carbonatés. Dans le même secteur, sont visibles de petites plages (2 à $3 \mathrm{~cm}$ de diamètre) de sables et de petits granules lavés.

D'autres zones d'enrichissement en carbonates sont visibles sur la coupe 2, au sommet de l'unité, et sur la coupe 5, vers la paroi calcaire (zone NE). Les carbonatations peuvent prendre la forme d'imprégnations matricielles, de concrétions racinaires ou de pseudomycéliums. On note qu'une structure lamellaire bien exprimée s'obsene dans la matrice de la partie inférieure de cette unité sédimentaire.

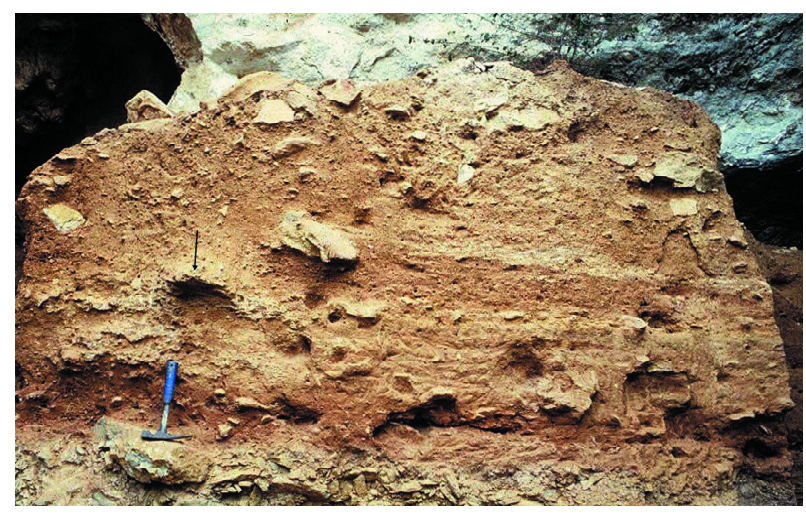

Figure 6 - Pech-de-l'Azé II. Vue de la partie supérieure de la coupe $n^{\circ} 2$. Noter l'organisation litée de la partie droite des dépôts. Le spéléothème incipient est indiqué par une flèche. (Epaisseur des dépôts : environ $2 \mathrm{~m}$ ).

Figure 6 - Pech-de-l'Azé II. View of the upper part of section 2. Note the bedded organization of deposits situated on the right part of the section. The incipient speleothem is shown by an arrow. (Thickness of deposits: ca $2 \mathrm{~m}$ )

\section{6 - DONNÉES ANALYTIQUES}

\subsection{Granulométrie}

Sur le diagramme triangulaire des textures (fig. 7), les points représentatifs des différentes unités sédimentaires sont, pour la plupart, regroupés près du pôle des sables. Les points qui s'en éloignent correspondent aux zones rouges observées au sein de l'unité 2 .

Les courbes cumulatives obtenues forment trois grandes familles morphologiques. Une première famille concerne les courbes représentatives de l'unité 1 (fig. 8). Elles sont unimodales et font apparaître le bon classement de ces sédiments sur les sables moyens ou sur les sables grossiers.

La deuxième famille (fig. 9) correspond aux sédiments non ou très faiblement rubéfiés des autres unités sédimentaires. Elles sont moins bien classées que les précédentes et montrent un mode principal sur les sables moyens ainsi qu'un mode secondaire atténué sur les limons fins et les argiles. La troisième famille (fig. 10) est représentative des sédiments rubéfiés des unités 2 et 4 . Elle est caractérisée par des courbes nettement bimodales comportant un premier mode sur les sables moyens et un second mode sur les limons fins et les argiles.

\section{2 - Micromorphologie}

Seules les unités 1, 2 et 4 ont fait l'objet d'analyses micromorphologiques. L'unité 3 , essentiellement composée de cailloux et de blocs associés à des sédiments fins interstitiels peu abondants et très peu cohérents, n'a pas été prélevée dans cette perspective. L'unité 1 , essentiellement sableuse, n'a pu être échantillonnée que dans les zones où elle était suffisamment consolidée par les carbonates.

\section{Unité 1}

Les sédiments (fig. 11) présentent une distribution $\mathrm{g} / \mathrm{f}$ (distribution des particules grossières par rapport aux fines) de type géfurique à chitonique. Le squelette est composé de sables bien classés, de diamètre moyen. II comprend principalement des quartz associés à de rares fragments osseux et à des grès ferrugineux. Les grains de quartz sont généralement arrondis et souvent creusés de golfes de corrosion. Des argiles ferruginisées brun foncé massives forment des revêtements et des ponts entre les grains du squelette. De la microsparite colmate partiellement les vides d'entassement. Quelques biotubules recoupent les sédiments.

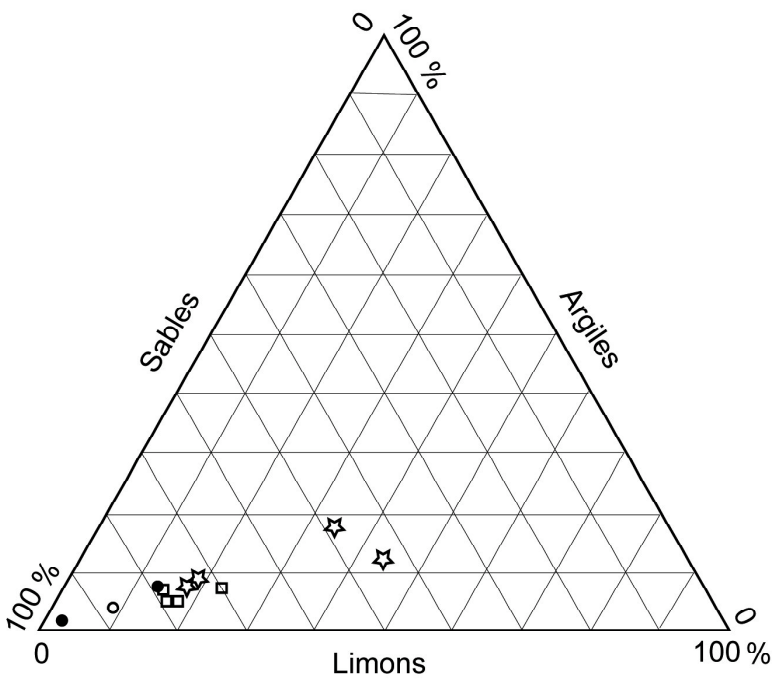

Figure 7 - Pech-de-l'Azé II. Diagramme triangulaire des textures. Cercles pleins : échantillons représentatifs de l'unité 1 - Cercle évidé : échantillon représentif de l'unité 3 - Etoiles : échantillons représentatifs de l'unité 2 - Carrés : échantillons représentatifs de l'unité 4.

Figure 7 - Pech-de-l'Azé II. Triangular diagram of textures. Full circles: samples representative of unit 1 - stars: samples representative of unit 2 - open circle: sample representative of unit 3 - squares: samples representative of unit 4. 

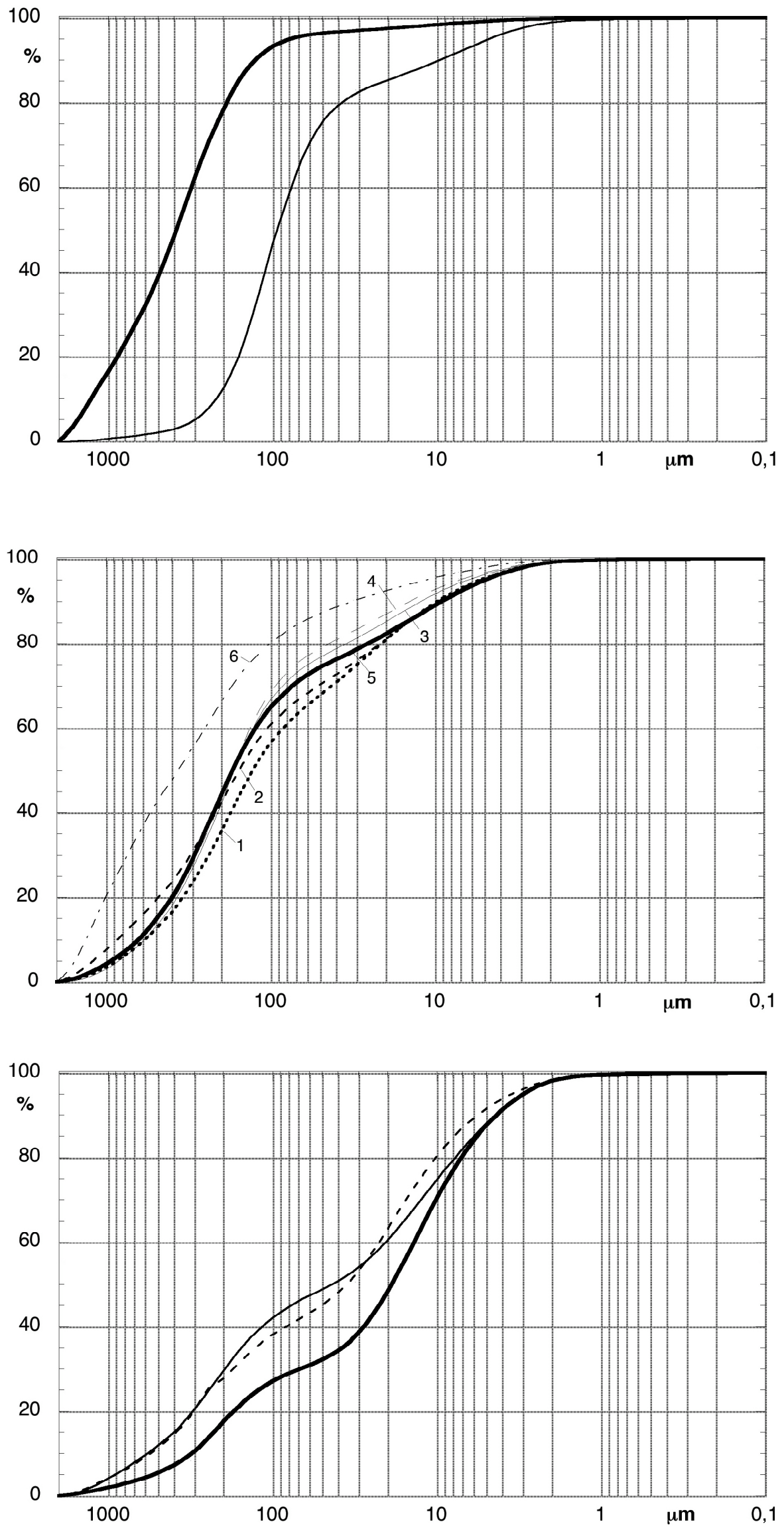

Figure 8 - Pech-de-l'Azé II. Courbes cumulatives représentatives de l'unité 1. Trait épais : sédiments grossiers - trait fin : sédiments fins

Figure 8 - Pech-de-l'Azé II. Cumulative curves representative of unit 1Bold line: coarse sediments - fine line: fine sediments.

Figure 9 - Pech-de-l'Azé II. Courbes cumulatives représentatives des sédiments non rubéfiés des unités 2, 3 et 4 . 1 à 4 : unité 4 ; 5 : unité 2 ; 6 : unité 3

Figure 9 - Pech-de-l'Azé II. Cumulative curves representatives of non-reddish sediments of units 2, 3 and 4 . 1 to 4 : unit 4 5 : unit 2 - 6 : unit 3

Figure 10 - Pech-de-l'Azé II. Courbes cumulatives représentatives des sédiments rubéfiés des unités 2 et 4. Trait fin : unité 2, coupe 2 - trait épais : unité 4 , coupe 5 - trait interrompu : unité 2 , coupe 5

Figure 10 - Pech-de-l'Azé II. Cumulative curves representative of reddish sediments of units 2 and 4. Fine line: unit 2, section 2 - bold line: unit 4, section 5 - dashed line: unit 2, section 5 


\section{Unité 2}

A la base de l'unité, les sédiments fins sont constitués de sables limoneux faiblement argileux. Les sables présentent un classement moyen à médiocre. Ils comprennent principalement des quartz auxquels s'ajoutent des grains phosphatés, des lithoclastes calcaires, des fragments de spéléothème et de pendants ainsi que quelques fragments de grès ferrugineux. Localement, ce matériel sédimentaire montre un litage net. II est en outre affecté par une structure lamellaire bien exprimée, triée ou non (Dumanski and St Arnaud 1966 ; Van Vliet Lanoë 1976). Cette structure lamellaire, localement recoupée par des biotubules, se superpose à une structure ovoïde préexistante (fig. 12). Des coiffes limono-argileuses litées se développent à la surface supérieure de certains grands clastes allongés ou forment des enrobements dissymétriques autour des grains du squelette et des agrégats granulaires (fig. 13).

Au sommet de l'unité, le sédiment comporte davantage de fines (limons et argiles) et présente une structure variable : granulaire, finement polyédrique ou lamellaire non triée (fig. 14 et 15). La composition du squelette est analogue à celle de la base de l'unité. Des coiffes limono-argileuses dissymétriques enrobent les clastes.

\section{Unité 4}

La partie inférieure de l'unité présente une structure lamellaire bien exprimée. Celle-ci est généralement de type trié, mais peut être également non triée dans les zones les plus argileuses. Les lamelles triées, d'épaisseur millimétrique, comportent une base sableuse et un sommet enrichi en argiles limoneuses brunes (fig. 16). Des coiffes limono-argileuses s'observent à la partie supérieure des clastes. Des biotubules peu abondants recoupent la structure lamellaire.

Outre les graviers calcaires, la fraction grossière du sédiment comprend en majorité des sables quartzeux de taille variable associés à des lithoclastes calcaires, des grains phosphatés, des fragments osseux et quelques fragments de spéléothème. Les grains phosphatés et les fragments osseux peuvent être localement abondants.

La partie supérieure de l'unité est très fortement bioturbée. La distribution des particules grossières par rapport aux fines («distribution $g / f »)$ est variable, généralement de type énaulique ou chitonique (fig. 17). Dans les vides d'entassement s'observent des agrégats fécaux de 30 à $50 \mathrm{~mm}$ de diamètre, de type enchytrée. Autour des grains du squelette et des graviers calcaires se développent fréquemment des revêtements argilo-organiques brun foncé, d'épaisseur irrégulière, formés d'agrégats plus ou moins fusionnés ou présentant un aspect massif (fig. 17). On note l'existence de feutrages micritiques et d'hypocutanes calcitiques respectivement dans et autour des biotubules (fig. 18).

Le squelette présente la même composition minéralogique que celle décrite pour la partie inférieure de l'unité. Là aussi, les grains phosphatés peuvent devenir très abondants dans certains niveaux. Une organisation litée est localement

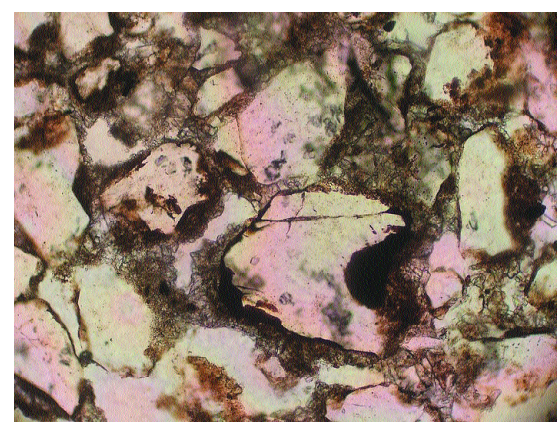

Figure 11 - Pech-de-l'Azé II. Microfaciès de l'unité 1 (LN). Des grains de quartz, parfois carriés, sont revêtus par des argiles ferrugineuses de couleur sombre. Les vides d'entassement sont partiellement colmatés par de la microsparite. (Largeur de la photo : $2 \mathrm{~mm}$ )

Figure 11 - Pech-de-l'Azé II. Microfacies of unit 1 (PPL). Quartz grains, coated by dark ferrugineous clays, are sometimes etched. The packing voids are partially infilled with microsparite. (Frame length: $2 \mathrm{~mm}$ )

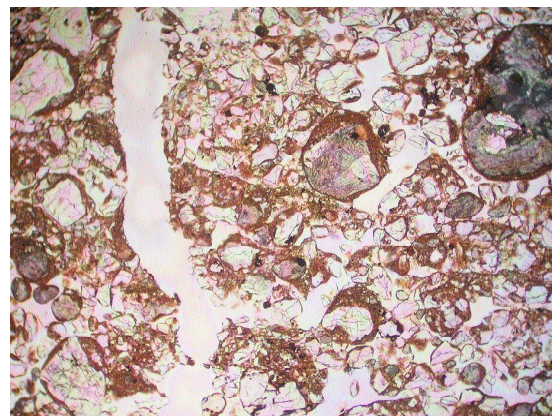

Figure 12 - Pech-de-l'Azé II, unité 2. Microphoto (LN). Une structurelamellaire recoupée par un biotubule se superpose à structure ovoïde. (Largeur de la photo : 5,3 $m m$ )

Figure 12 - Pech-de-l'Azé II, unit 2. Photomicrograph (PPL). A platy structurecrosscut by a biotubule is superimposed on an ovoïd structure (Frame length: $5.3 \mathrm{~mm}$ )

conservée. Elle se matérialise par des lits de fines intercalées dans un matériel sableux ou par des lits de grains phosphatés de la taille des sables grossiers alternant avec des lits composés de grains de quartz plus petits. 


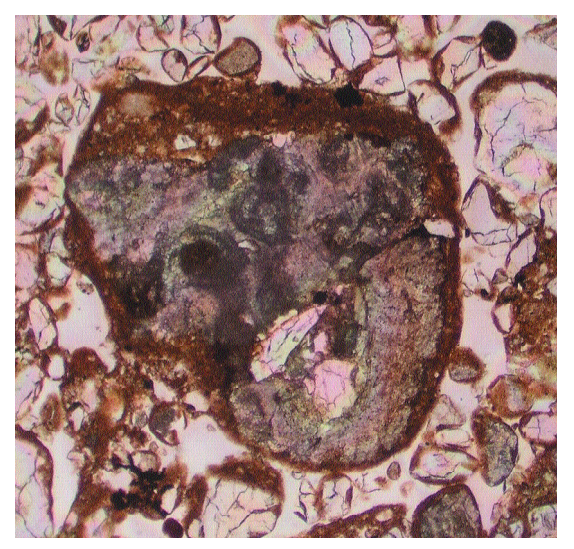

Figure 13 - Pech-de-l'Azé II, unité 2. Microphoto (LN). Une coiffe dissymétrique se développe autour d'un lithoclaste calcaire. (Largeur de la photo : 1,6 mm)

Figure 13 - Pech-de-l'Azé II, unit 2. Photomicrograph (PPL). A asymetric coating developed around a calcareous lithoclast (Frame length: $1.6 \mathrm{~mm}$ )

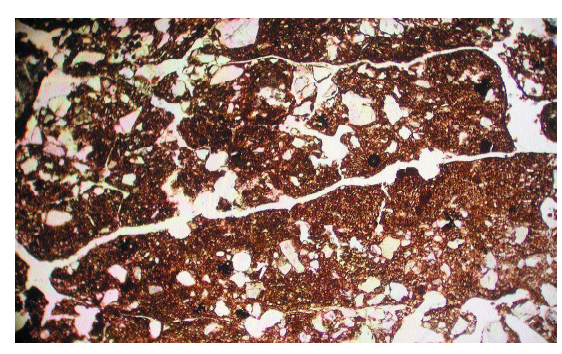

Figure 14 - Pech-de-l'Azé II, unité 2. Microphoto (LN). Une structure lamellaire non triée se développe dans les zones les plus riches en argile. (Largeur de la photo : 3,5 mm)

Figure 14 - Pech-de-l'Azé II, 2. Photomicrograph (PPL). A non sorted platy structure developed in the more clayey parts of the unit. (Frame length: 3,5 mm)

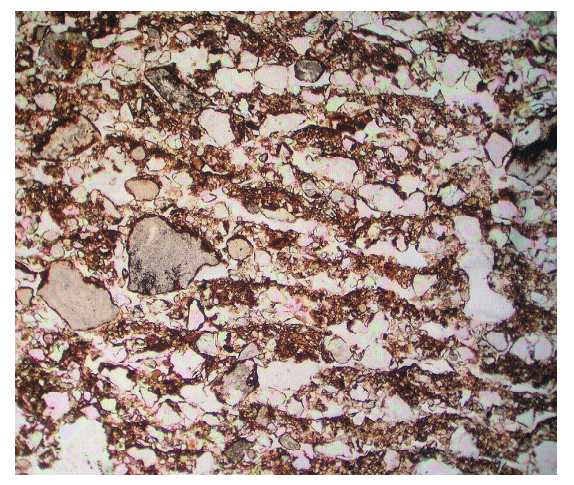

Figure 15 - Pech-de-l'Azé II, unité 4. Microphoto (LN). Structurelamellaire triée. (Largeur de la photo : 6,3 $\mathrm{mm}$ )

Figure 15 - Pech-de-l'Azé II, unit 4. Photomicrograph (PPL). Sorted platy structure(Frame length: 6,3 $\mathrm{mm}$ )

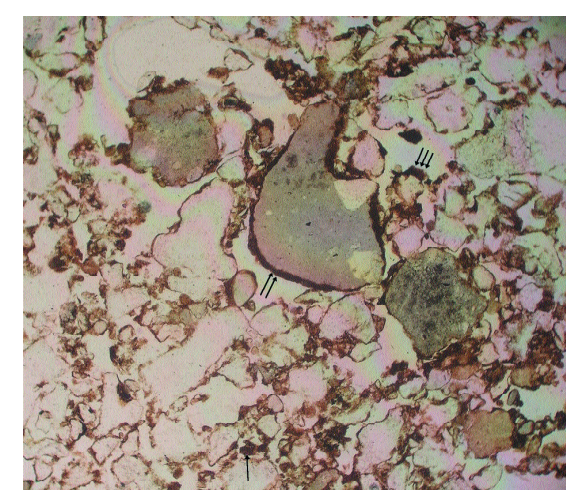

Figure 16 - Pech-de-l'Azé II, unité 4. Microphoto (LN). Des agrégats fécaux de type enchytrée s'observent dans les vides d'entassements (flèche). Des revêtements argiloorganiques irréguliers se développent à la surface des grains sableux (double flèche) et sur les parois des biotubules (triple flèche). (Largeur de la photo : 2, $4 \mathrm{~mm}$ )

Figure 16 - Pech-de-l'Azé II. Photomicrograph of unit 4 (PPL). Fecal aggregates of enchytraeid type are observed in packing voids (arrow). Irregular organic clayey coatings made of more or less coalescent aggregates develop on the surface of sand grains (double arrow) and on the walls of biologic voids (triple arrow). (Frame length: $2.4 \mathrm{~mm}$ )

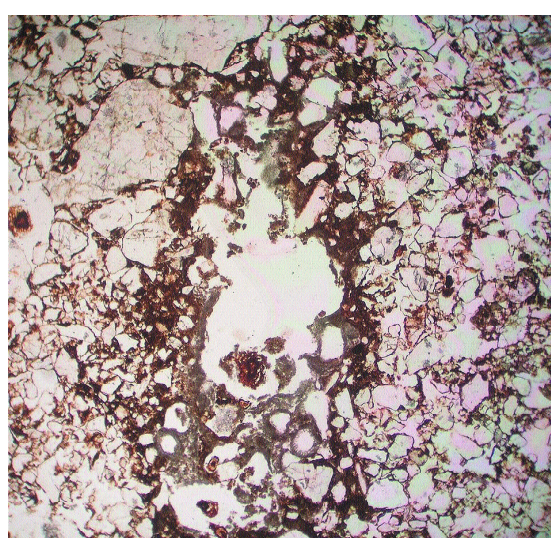

Figure 17 - Pech-de-l'Azé II, unité 4. Microphoto (LN). On observe un feutrage micritique dans la lumière d'un vide racinaireet un hypocutane calcitique autour de ce demier. (Largeur de la photo : $5 \mathrm{~mm}$ )

Figure 17 - Pech-de-l'Azé II, unit 4. Photomicrograph $(P P L)$. We observe a micritic felting in a root void and, around this one, a calcitic hypocoating. (Frame length: $5 \mathrm{~mm}$ ) 


\section{7 - INTERPRÉTATION MORPHO-DYNAMIQUE ET DIAGÉNÉTIQUE}

Sur la base des études de terrain et des résultats analytiques obtenus, il est possible de décrire un certain nombre de phases évolutives de la cavité (tabl. 2).

La première phase comespond à la formation de la cavité. Il est difficile de dire si celle-ci résulte d'une évolution en contexte vadose ou en contexte phréatique. Néanmoins, un certain nombre de poches, encore visibles au toit de la cavité et susceptibles de représenter des cupules de dissolution, amènent à penser que ce réseau s'est sans doute constitué en milieu phréatique (Bretz 1942 ; White 1988). Une telle hypothèse implique un niveau de base local situé au-dessus de la cavité actuelle.

La deuxième phase est caractérisée par le dépôt de sables fluviatiles (unité 1) en liaison avec le fonctionnement d'un cours d'eau souterrain. Le caractère fluviatile de ces sables est inféré à partir de leur organisation litée (litage oblique et entrecroisé) et de leur bon classement mis en évidence à la fois par les analyses granulométriques et l'obsenation microscopique. Cet épisode morphogénique implique un abaissement sensible du niveau de base local, au-dessous de celui de la cavité. Les structures sédimentaires observées dans la partie la plus interne de la cavité (coupe 7) témoignent d'un écoulement vers le NO, c'est-àdire vers l'ouverture actuelle. En revanche, les lits obliques dessinés par Laville (1973) au sommet de sa coupe 6, indiquent un écoulement de sens opposé (i.e. vers le SE). Ce constat est sans doute à mettre sur le compte d'un système d'écoulement complexe, peut-être en liaison avec des pertes. Les autres affleurements actuellement visibles à la base des coupes 2,3 , 4 et 5 sont d'épaisseur très réduite et ne permettent pas de clarifier cette question.

La composition pétrographique de ces alluvions (quartz, grès ferrugineux, graviers de silice) et l'aspect corrodé de nombreux grains de quartz indiquent que ces sédiments proviennent pour l'essentiel d'une reprise des altérites qui recouvrent le plateau. Les revêtements argilo-ferrugineux obsenés sous le microscope sont probablement en partie hérités de ces formations originelles. Une autre partie (i.e. les ponts entre les grains) est sans doute liée à des perc olations d'eaux chargées en fines.

La microsparite qui consolide localement ces dépôts s'est déposée en contexte vadose. Elle correspond probablement à un phénomène diagénétique tardif car elle n'est pas affectée par le cryosol qui se développe dans les dépôts sus-jacents. En revanche, les spéléothèmes, retrouvés à l'état de fragments dans les unités 2 et 3 , se sont probablement formés lors de cette phase sur le plafond et les parois de la cavité.

La troisième phase comespond au dépôt de l'unité sédimentaire 2 au cours de laquelle la grotte poursuit son évolution en contexte vadose. L'organisation générale des sédiments de cette unité peut être interprétée soit comme le résultat d'écoulements en masse accompagnés de ruissellements, soit comme celui de ruissellements associés à une éboulisation modérée. La seconde hypothèse permet cependant de mieux tenir compte des différentes caractéristiques de cette unité. En effet, le ruissellement y est attesté par la présence de lits granoclassés observés sur les différentes coupes ainsi qu'à l'échelle microscopique. En outre, l'absence d'une structure litée généralisée est fréquente dans ce type de dépôt (Bertran et Texier 1997 ; Lenoble 2005). Elle s'explique par l'action de multiples facteurs associés au phénomène de ruissellement : mauvais classement dû aux faibles tranches d'eau impliquées dans les écoulements turbulents (Moss et Walker 1978 ; Raws 1987 ; Scoging 1989), fréquence des écoulements hyperconcentrés (Bertran et Texier 1999), éboulement de berges de rigoles (De Ploey 1974 ; Govers 1987), développement de petites coulées boueuses localisées (De Ploey 1971, 1974 ; De Ploey et Moeyerson 1975 ; Gerits et al. 1987), phénomène de «piping» (Govers 1987 ; Campbell 1989), action des phénomènes diagénétiques (bioturbation, précipitation de carbonates,...) (Bertran et Texier 1997 et 1999), action concomitante du gel (Lenoble 2005). Ces dépôts non lités correspondent aux zones où la sédimentation est normalement faible (Bertran et Texier 1999 ; Lenoble 2005). On notera également que ce phénomène de ruissellement permet de rendre compte de l'émoussé très accentué présenté par les fragments rocheux contenus dans cette unité sédimentaire.

La plus ou moins grande abondance des cailloux et des blocs calcaires s'explique par le fait que l'activité de l'éboulisation a varié à la fois dans l'espace (i.e. selon les secteurs de la cavité) et dans le temps. En effet, il n'existe pas dans l'unité 2 de niveaux caillouteux spécifiques pouvant être suivis sur toutes les coupes. De plus, l'absence d'orientation des clastes et/ou de macrostructures caractéristiques ne permet pas de retenir l'hypothèse d'une mise en place dominée par la solifluxion (Bertran et al. 1995).

Les variations de couleurs constatées sont vraisemblablement le résultat à la fois de phénomènes synsédimentaires (différences de proportions entre sédiments provenant des altérites et sédiments dérivant de la désagrégation du calcaire encaissant, plus ou moins forte teneur en matière organique) et de phénomènes diagénétiques (imprégnations carbonatées). L'existence de sols rouges lessivés avancée par Laville et attribués aux Interstades Riss I Riss II et Riss II - Riss III (Laville 1973), ne peut être maintenue. En effet, ces niveaux rouges ne présentent pas de continuité latérale et, comme l'avait déjà montré Goldberg (1979), ne comportent aucun trait caractéristique des luvisols (i.e. revêtements argileux in situ). De plus, la présence de fragments de spéléothèmes dans les coupes 1 (cf. Laville 1973) et 2 (cf. supra) indiquent que l'unité 2 s'est déposée à l'intérieur de la cavité alors que l'ouverture de celle-ci se situait probablement à l'ouest de l'actuelle.

Les phénomènes sédimentaires inférés pour la mise en place de l'unité 2 ne permettent pas de décrire une ambiance climatique très spécifique. En effet, le ruissellement est un mécanisme susceptible de se manifester dans une gamme climatique très large (surtout en grotte). En outre, l'éboulisation y est globalement peu active et peut simplement s'expliquer par des phénomènes de fatigue ou de détente des parois (Renault 1967 ; Whalley 1984) ainsi que par des phénomènes d'ajustement de la voûte à un profil d'équilibre plus stable (White 1988). 


\begin{tabular}{|c|c|c|c|c|}
\hline \multicolumn{2}{|c|}{$\begin{array}{c}\text { Unités } \\
\text { stratigr. }\end{array}$} & $\begin{array}{l}\text { Evolution morpho- } \\
\text { sédimentaire }\end{array}$ & $\begin{array}{c}\text { Datations } \\
\text { numériques }\end{array}$ & $\begin{array}{l}\text { Propositions } \\
\text { chronologiques }\end{array}$ \\
\hline I & l & $\begin{array}{l}\text { Phase 7: } \\
\text { - } \text { Effondrement de la partie externe } \\
\text { du toit de la cavité } \\
\text { - } \text { Carbonations } \\
\text { - } \text { Bioturbations }\end{array}$ & I & $\begin{array}{c}\text { OIS } 1 \\
\text { (Holocène) }\end{array}$ \\
\hline I & l & $\begin{array}{l}\text { Phase } 6 \text { : } \\
\begin{array}{l}\text { - Formation d'un gélisol profond ou } \\
\text { d'un pergélisol }\end{array}\end{array}$ & I & OIS 2 ou/et 4 \\
\hline $\begin{array}{l}2 \\
\text { à } \\
4\end{array}$ & 4 & $\begin{array}{l}\text { Phase } 5 \text { : } \\
\text { - } \text { Ruissellement et éboulisation } \\
\text { modérée } \\
\text { - } \text { Formation localisée de } \\
\text { concrétions carbonatées et de } \\
\text { spéléothèmes }\end{array}$ & $\begin{array}{c}41 \pm 6 \\
\text { à } 78 \pm 6,6 \mathrm{Ka}^{*} \\
103 \pm 14 \mathrm{Ka}^{* *}\end{array}$ & OIS 5 \\
\hline $\begin{array}{l}5 \\
\text { et } \\
x\end{array}$ & 3 & $\begin{array}{l}\text { Phase 4: } \\
\text { - } \text { Eboulisation très active } \\
\text { - } \quad \text { Formation d'un gélisol profond ou } \\
\text { d'un pergélisol } \\
\text { - } \quad \text { Cryoturbation localisée }\end{array}$ & $\begin{array}{c}124 \pm 10 \\
\text { à } \\
154 \pm 14 \mathrm{Ka}^{*}\end{array}$ & $\begin{array}{l}\text { Partie supérieure de } \\
\text { I'OIS } 6\end{array}$ \\
\hline $\begin{array}{l}6 \\
\text { à } \\
9\end{array}$ & 2 & $\begin{array}{l}\text { Phase 3: } \\
\text { - } \text { Ruissellement et éboulisation } \\
\text { modérée } \\
\text { - Formation d'un gélisol peu } \\
\quad \text { profond }\end{array}$ & $\begin{array}{c}117 \pm 13 \\
\text { à } \\
175 \pm 27 \mathrm{Ka}^{*}\end{array}$ & $\begin{array}{l}\text { Partie inférieure de } \\
\text { I'OIS } 6\end{array}$ \\
\hline 10 & 1 & $\begin{array}{l}\text { Phase 2: } \\
\text { - Accumulation de dépôts fluviatiles } \\
\text { endokarstiques } \\
\text { - Formation locale de } \\
\quad \text { spéléothèmes }\end{array}$ & I & $\begin{array}{c}\text { Fin : Stade } 7 \text { ? } \\
\text { Début: ? }\end{array}$ \\
\hline I & 1 & $\begin{array}{l}\text { Phase } 1 \text { : } \\
\text { - Formation du réseau karstique en } \\
\text { contexte phréatique }\end{array}$ & I & $?$ \\
\hline
\end{tabular}

* Dates ESR (Grün and Stringer 1991 ; Grün et al. 1991) : les dates mentionnées ici sont des dates E.U. ("Early Uptake") car elles sont jugées meilleures par les auteurs.

** Dates Th / U (Schwarcz and Blackwell 1983).

Tableau 2 - tableau synoptique montrant:

1) le découpage stratigraphique des dépôts du Pech-de-l'Azé Il selon H. Laville (HL) et selon J.P. Texier (JPT),

2) les principaux événements morpho-sédimentaires enregistrés dans le site,

3) les datations numériques actuellement disponibles et, 4) l'hypothèse chronologique proposée dans cet article. (OIS :

Stade isotopique de l'oxygène)

Table 2 - Synoptic table showing:

1) the stratigraphic divisions of Pech-de-l'Azé Il deposits following H. Laville (HL) and J.P. Texier (JPT),

2) the main morpho-sedimentary events recorded int the site,

3) the available numerical dates and, 4) the chronological hypothesis proposed in this paper. (OIS : Oxygen isotopic stage) 
Cependant, la structure granulaire de type ovoïde observée à l'échelle microscopique indique qu'un gélisol peu profond a accompagné la formation de ces dépôts. En effet, de telles structures se rencontrent habituellement dans les horizons de surface soumis à de nombreuses alternances gel-dégel (Bertran 1993). Elles témoignent de contraintes internes, responsables de la déformation et de la rotation des agrégats cryogéniques (Van Vliet Lanoë et al. 1984 ; Harris and Cook 1988).

La structure lamellaire de la matrice qui témoigne du développement d'un gélisol profond ou d'un pergélisol (Van Vliet Lanoë 1988), semble avoir été acquise postérieurement à la mise en place des dépôts (cf. infra). La présence d'une structure lamellaire non triée au sommet de l'unité s'explique par la plus grande richesse en argiles de cette zone. En effet, plusieurs auteurs (e.g. Mermut and St Arnaud 1981 ; Van Vliet Lanoë 1985) ont souligné que les structures triées s'exprimaient essentiellement dans les sédiments à dominante sableuse ayant une teneur en argile inférieure à $15 \%$. D'ailleurs, les deux types de structure (lamellaire triée et lamellaire non triée) coexistent dans la partie inférieure de l'unité et leur développement est visiblement lié à la texture plus ou moins argileuse des sédiments.

La quatrième phase évolutive comespond, pour l'essentiel, à un épisode d'éboulisation généralisé à l'ensemble de la cavité avec, pour corollaire, la mise en place de l'unité sédimentaire 3. Les phénomènes évoqués pour la phase précédente (détente, fatigue, ajustement de la voûte) ont sans doute joué un rôle dans la fragmentation des parois, notamment dans le détachement des grandes dalles calcaires observées sur la coupe 4. Cependant, plusieurs éléments permettent de penser que le gel a eu un rôle majeur dans la formation de cette unité :

1) la morphologie des fragments rocheux (plaquettes), très différente de celle des cailloux et blocs présents dans les unités sus- et sous-jacentes, évoque l'intervention d'un front de gel pénétrant parallèlement aux parois de la cavité et débitant celles-ci en écailles identiques à celles formées actuellement en milieu actif dans les calcaires massifs :

2) la généralisation du phénomène à l'ensemble de la cavité suggère l'intervention de facteurs extrinsèques de type climatique ;

3) les grandes dalles issues de la voûte sont ellesmêmes découpées en écailles parallèles à leur surface. Lors de cet épisode, les écoulements semblent s'être réduits de façon drastique. Seuls les sables qui colmatent plus ou moins les vides, témoignent de percolations locales, sans doute épisodiques, chargées en produits détritiques. Leur coloration variable est probablement à mettre en liaison avec l'origine des sédiments : «sidérolithique» pour les sables à dominante rouge, désagrégation du calcaire encaissant pour les sables à dominante jaune. Cette dernière proposition est notamment étayée par les résultats granulométriques exposés plus haut. Les sédiments rouges sont en effet plus argileux que les sédiments de couleur claire et présentent une texture voisine de celle des altérites qui recouvrent les plateaux de la région.
Le gel évoqué pour la genèse des fragments rocheux est également responsable de la structuration en lamelles de la matrice de cette unité (unité 3 ) ainsi que de celle de l'unité sous-jacente (unité 2).

Cette phase évolutive, fortement marquée par les phénomènes cryogéniques, serait donc en liaison avec une importante dégradation climatique au cours de laquelle le gel occasionne une desquamation très active des parois de la cavité ainsi que la formation d'un gélisol profond (voire d'un pergélisol) dans les dépôts sousjacents. Les involutions observées dans l'unité 3 s'interprètent comme des cryoturbations et procèdent du même phénomène.

La présence de fragments de spéléothème au niveau de la coupe 2 indique que l'ouverture de la grotte se situait toujours à l'ouest de l'actuelle.

La phase suivante (phase 5) comespond au dépôt de l'unité sédimentaire supérieure (unité 4). La sédimentogenèse est à nouveau dominée par le ruissellement associé à des chutes modérées d'éboulis. Les lithofaciès présents dans cette unité sont en effet fondamentalement les mêmes que ceux observés dans l'unité 2 : niveaux lenticulaires lités et granoclassés, intercalés dans des sables massifs et présence d'éboulis émoussés plus ou moins abondants selon les secteurs de la cavité. Le niveau à petits cailloux et granules arrondis qui débute cette unité peut être interprété comme un niveau de résidualisation, également lié au phénomène de ruissellement.

Les variations de couleurs des sédiments sont également à mettre sur le compte soit d'un héritage ("Sidérolithique» vs désagrégation du calcaire encaissant), soit d'une diagénèse (carbonatation, phosphatisation, imprégnation par des matières organiques).

Les concrétions carbonatées nodulaires de même l'amorce de plancher stalagmitique, visibles sur la coupe 2 au sein de cette unité (fig. 4 et 6), sont liées à des phénomènes d'égouttement, ce qui implique que cette partie du site était alors encore recouverte par un toit. Les petites plages de sables lavés et de granules observés dans la même zone témoignent d'un phénomène identique et marquent l'emplacement d'anciens trous de stillation. D'une façon plus générale, on constate que ce secteur a été, tout au long de l'histoire de la cavité, une zone où des suintements importants se sont produits. Ceci explique non seulement la formation localisée de spéléothèmes et de concrétionnements, mais aussi probablement l'effacement du litage et l'aspect massif des sédiments de cette zone.

La phase 6 qui succède, est caractérisée par le développement d'un cryosol profond, voire d'un pergélisol, comme en témoigne la structure lamellaire conservée dans la partie inférieure de l'unité 4 . La très forte polarisation des coiffes à la partie supérieure des lithoclastes et la bonne conservation des structures sédimentaires (litage) impliquent en effet que cet épisode froid est postérieur à la mise en place des dépôts. Dans la partie supérieure de l'unité, les traits cryogéniques ont probablement été effacés à la suite du dernier épisode diagénétique subi par cette série sédimentaire (phase 7 , cf. infra). 
La phase 7 représente la phase d'évolution ultime du site. Elle est caractérisée par un effondrement du toit de la cavité, comme en témoignent les gros blocs plus ou moins jointifs qui surmontent les dépôts de la coupe 5 (fig. 5), par une bioturbation très importante du sommet des dépôts et par des concrétionnements carbonatés variés : concrétions racinaires, pseudomycéliums, imprégnations. Ces demières affectent non seulement le sommet des dépôts, mais aussi l'ensemble des unités au voisinage de la paroi rocheuse (coupe 5, fig. 5). A l'échelle microscopique, les feutrages micritiques localisés dans les biotubules s'interprètent comme le résultat d'une biominéralisation de filaments de champignons saprophytes développés autour des racines (Verrecchia and Verrecchia 1994). Les hypocutanes calcitiques, associés aux traits précédents, sont probablement dus à une précipitation rapide de carbonate de calcium par suite d'une dessiccation locale des sédiments provoquée par le métabolisme des plantes (Wieder and Yaalon 1982 ; Becze-Deak et al. 1997). La bioturbation se manifeste par le développement de nombreux tubules et par d'abondants agrégats fécaux de type collembole ou enchytrée, parfois redistribués par les percolations autour des grains du squelette pour former des revêtements plus ou moins continus.

\section{8 - REMARQUES SUR LA CHRONOLOGIE DES DIFFÉRENTES PHASES ÉVOLUTIVES (tabl. 2)}

Les propositions faites ci-dessous sont basées sur une tentative "d'harmonisation» entre, d'une part, les dates numériques obtenues (Schwarcz et Blackwell 1983 ; Grün et Stringer 1991; Grün et al. 1991) et, d'autre part, les résultats géologiques présentés plus haut.

Mises à part quelques dates attribuables au début du stade isotopique de l'oxygène 5 (OIS 5 ), la plupart des dates ESR obtenues dans les niveaux $5,6,7,8$ et 9 de $\mathrm{H}$. Laville (= unités 2 et 3 ) se situent à l'intérieur de l'OIS 6 (Grün et Stringer 1991 ; Grün et al. 1991) (fig. 1). II est donc logique de penser que la mise en place de ces unités s'est effectuée au cours de cet épisode climatique. Dans cette hypothèse, l'unité 2 qui traduit un froid modéré (3ème phase évolutive), pourrait être mise en relation avec la première partie de ce stade tandis que la phase suivante, beaucoup plus froide (cf. supra), en représenterait la fin. On notera qu'une évolution climatique globale identique a été mise en évidence à l'échelle régionale par les faunes de grands mammifères (Delpech et Prat 1995).

Aucun des processus sédimentaires identifiés dans l'unité 4 n'est caractéristique d'un environnement froid. La présence d'un plancher stalagmitique incipient formé in situ laisse envisager un climat contemporain relativement tempéré. En effet, ce type de concrétion a tendance à se former plutôt durant les phases interglaciaires ou interstadiaires (Baker et al. 1993 ; Gascoyne et Ford 1984 ; Onac et Lauritzen 1996). C'est pourquoi, malgré les dates ESR contemporaines des OIS 4 et 3 obtenues dans cette unité (fig. 1), on privilégiera l'hypothèse de dépôts formés au cours l'OIS 5. Cette hypothèse se trouve par ailleurs en bon accord avec la date Th/U de $103_{-25}^{+30} \mathrm{Ka}$ (Schwarcz et
Blackwell 1983) fournie par le spéléothème que nous venons d'évoquer ainsi qu'avec un certain nombre de dates ESR (fig. 1).

Le gélisol profond ou le pergélisol qui succède au dépôt de l'unité 4 (phase évolutive 6) s'est probablement développé au cours de l'OIS 4 ou de l'OIS 2. Les connaissances acquises dans le nord de l'Aquitaine montrent en effet que ces deux épisodes ont pu générer de telles structures c ryogéniques (Bertran et Texier 1990 ; Texier et Bertran 1993 ; Texier 1996 ; Texier 2003b).

La phase évolutive finale, caractérisée par l'effondrement de l'avant de la cavité, une bioturbation très active et des phénomènes de concrétionnement variés, se rapporte logiquement à l'Holocène (OIS 1).

II n'est actuellement pas possible de situer chronologiquement les deux premiers stades évolutifs inscrits dans ce site (Creusement du conduit karstique et dépôt des sables fluviatiles). On soulignera néanmoins qu'ils représentent vraisemblablement des durées relativement longues. La date de $240 \pm 30 \mathrm{Ka}$ obtenue sur les fragments de spéléothèmes contenus dans l'unité 3 (= couche 5 de Laville) (Schwarcz et Blackwell 1983) laisse supposer que la phase évolutive 2 s'est vraisemblablement achevée au cours de I'OIS 7, si on admet que ces concrétions se sont bien formées sur la paroi de la cavité lors de cet épisode morphologique (cf. paragraphe 7).

On soulignera enfin que le "calage» chronologique proposé ici pour les phases évolutives 3,4 et 5 (mise en place des unités 2, 3 et 4) est en bon accord avec celui avancé par $\mathrm{F}$. Delpech à partir des biozones définies en Aquitaine septentrionale (Delpech 1999 in : Texier et al. 1999).

\section{9 - CONCLUSIONS}

Le site du Pech-de-l'Azé II a enregistré sept principales phases morpho-sédimentaires. La première correspond au creusement de la cavité, probablement en contexte phréatique. La deuxième voit la mise en place de dépôts fluviatiles endokarstiques (Unité sédimentaire 1). L'épisode suivant (phase 3 ), au cours duquel se manifeste un froid modéré (gélisol), est caractérisé par l'action du ruissellement accompagné par une éboulisation peu active ; l'unité 2 se met alors en place. Lors de la phase 4, l'éboulisation devient prédominante, les écoulements sont réduits et le froid s'intensifie ; un gélisol profond ou un pergélisol se développe dans les unités 2 et 3 et des phénomènes de c nyoturbation affectent localement l'unité 3 . Le dépôt de l'unité 4 (phase 5 ) résulte de l'action prédominante du ruissellement associé à une éboulisation modérée ; des concrétionnements carbonatés (nodules, spéléothèmes) se forment localement en liaison avec des phénomènes d'égouttement. A la fin de cet épisode, la cavité est presque complètement colmatée. Les phases suivantes comespondent essentiellement à une évolution diagénétique des dépôts : d'abord, formation d'un gélisol profond ou d'un pergélisol (phase 6) puis, développement d'une 
bioturbation très active et précipitation de concrétions carbonatées (phase 7). C'est également au cours de cette phase ultime que se fragmente et s'effondre la partie la plus externe du toit de la cavité.

En s'appuyant sur les datations numériques disponibles (Grün et Stringer 1991 ; Grün et al. 1991 ; Schwarcz et Blackwell 1983), il est possible de situer chronologique ment certaines de ces phases évolutives : les phases 3 et 4 seraient contemporaines respectivement du début et de la fin du stade isotopique 6 , la phase 5 du stade 5 , la phase 6 du stade 4 ou 2, la phase 7 de l'Holocène.

Parmi les mécanismes sédimentaires identifiés, le ruissel lement joue un rôle prédominant dans toute l'épaisseur du remplissage (excepté dans l'unité 3). Or, ce processus est potentiellement très perturbateur vis-à-vis des niveaux d'occupations préhistoriques (Lenoble 2005). Ceci explique probablement en partie l'incohérence apparente et le groupement des dates ESR obtenues au sein des unités 2 et 3 d'une part, et 4 d'autre part (Texier 2001a). Aussi, dans l'état actuel de nos connaissances et en l'absence d'étude géoarchéologique approfondie, il semble plus prudent de considérer les objets archéologiques contenus dans ces unités comme des ensembles cumulant les données d'une période de temps relativement longue que de se référer à des niveaux plus minces dont la pertinence n'est pas certaine.

\section{Remerciements}

Ces travaux ont été réalisés dans le cadre du PCR «Bio- et litho-stratigraphie de gisements paléolithiques de référence du Périgord" financé par des crédits du Ministère de la Culture et de la Communication. Je remercie également les deux rapporteurs de cet article, P. Goldberg et J.P. Lautridou, pour leurs remarques constructives.

\section{BIBLIOGRAPHIE}

BAKER A., SMART P. L., FORD D. C. 1993 - Northwest European palaeoclimate as indicated by growth frequency variations of secondary calcite deposits. Palaeogeography, Palaeoclimatology, Palaeoecology, 100 (3) : 291-301.

BECZE-DEAK J., LANGHOR R. and VERRECCHIA E.P. 1997 - Small scale secondary $\mathrm{CaCO} 3$ accumulations in selected sections of the European loess belt. Morphological forms and potential for paleoenvironmental reconstruction. Geoderma, 76 : 221-250.

BERTRAN P. 1993 - Deformation-induced microstructures in soils affected by mass movements. Earth Surface Processes and Landforms, 18 : 645-660.

BERTRAN P. et TEXIER J.P. 1990 - L'enregistrement des phénomènes pédo-sédimentaires et climatiques dans les dépôts colluviaux d'Aquitaine : l'exemple de la coupe des Tares (Dordogne). Quatemaire, 1(1) : 77-91.
BERTRAN P. et TEXIER J.P. 1997 - Géoarchéologie des versants. In «Dynamique des paysages», J.P. Bravard et M. Prestreau Ed., Documents d'Archéologie en Rhône-Alpes, p. 59-86.

BERTRAN P. and TEXIER J.P. 1999 - Facies and microfacies of slope deposits. Catena, $35:$ 99-121.

BERTRAN P., FRANCOU B. et TEXIER J.P. 1995 - Stratified slope deposits : the stone-banked sheets and lobes model. In : O. Slaymaker Ed.,„Steepland Geomorphology», Wiley, p. 147-169.

BINFORD L.F. and BINFORD S.R. 1966 - A preliminary analysis of functional variability in the Mousterian of Levallois facies. American Anthropologist, 68(2), p. 238-295.

BINFORD L.F. and BINFORD S.R. 1969 - Stone tools and human behaviour. Scientific American, 220(4), p. 70-84.

BORDES F. 1953 - Essai de classification des industries moustériennes. Bulletin de la Société Préhistorique de France, 50, p. 457-466.

BORDES F. 1954-1955 - Les gisements du Pech-de-l'Azé (Dordogne). 1 Le Moustérien de tradition acheuléenne. L'Anthropologie, 6, p. 205-244.

BORDES F. 1971 - Observations sur l'Acheuléen des grottes de Dordogne. Munibe, 23, p. 5-24.

BORDES F. 1981 - Vingt-cinq ans après : le complexe moustérien revisité. Bulletin de la Société Préhistorique de France, 78(3), p. 77-87.

BORDES F. et BOURGON M. 1951 - Le gisement du Pech-de-l'Azé Nord. Campagne 1950-1951. Les couches inférieures à Rhinoceros Merki. Bulletin de la Société Préhistorique de France, 48, p. 520-538.

BORDES F. et PRAT F. 1965 - Observations sur les faunes du Riss et du Würm I. L'Anthropologie, 69(1-2), p. 31-46.

BRETZ J.H. 1942 - Vadose and phreatic features of limestone caverns. The Journal of Geology, 50 (6) : 675-811.

BULLOCH P., FEDOROFF N., JONGERIUS A., STOOPS G., TURSINA T. and BABEL U. 1985 - Handbook for soil thin section description. Waine Research Publications, $152 \mathrm{p}$.

CAMPBELL I.A. 1989 - Badlands and badland gullies.In : D.S.G. Thomas Ed., «Arid Zone Geomorphology», p. 159-183.

DELPECH F. et PRAT F. 1995 - Nouvelles observations sur les faunes acheuléennes de Combe-Grenal (Domme, Dordogne). Paléo, 7 : 123-137.

DE PLOEY J. 1971 - Liquefaction and rainwash erosion. Zeitschrift für Geomorphologie, 4 : 491-496. 
DE PLOEY J. 1974 - Mechanical properties of hillslopes and their relation to gullying in Central semiarid Tunisia. Zeitschrift für Geomorphologie N.F., 21 : 177-190.

DE PLOEY J. and MOEYERSONS J. 1975 - Runoff creep of coarse debris : experimental data and some field observations. Catena, 2 : 275-288.

DIBBLE H. 1987 - Reduction sequences in the manufacture of mousterian implements of France. In : O. Soffer (ed.), The Pleistocene of Old World: regional perspectives, New York, Plemum, p. 33-45.

DIBBLE H. and MELLARS P. 1992 - The Middle Paleolithic; adaptation, behavior and variability. The University Museum, University of Pennsylvania, University Museum Series I, $461 \mathrm{p}$.

DUMANSKI J. and ST-ARNAUD R.S. 1966 - A micropedological study of eluvial soil horizons. Canadian Journal of Soil Science, 46 (1) : 287-292.

GASCOYNE M. and FORD D. C. 1984 - Uranium series dating of speleothems, Part II. Results from the Yorkshire Dales and implications for cave development and quaternary climates. Cave Science, 11 (2) : 65-85.

GERITS J., IMESON A.C., VERSTRATEN J.M., BRYAN R.B. 1987 - Rill development and badland regolith properties. Catena-Supplement $8: 141-160$.

GOLDBERG P. 1979 - Micromorphology of Pech-del'Azé II sediments. Jo u mal of Archaeological Science, 6 : 17-47.

GOVERS G. 1987 - Spatial and temporal variability in rill development processes at the Huldenberg experimental site. Catena Supplement 8 : 17-34.

GRUN R. and STRINGER C.B. 1991 - Electro Spin Resonance dating and the evolution of modern humans. Archaeometry, 33 (2) : 153-199.

GRUN R., MELLARS P. and LAVILLE H. 1991 - ESR chronology of a 100,000-year archaeological sequence at Pech de l'Azé II, France. Antiquity, 65 : 544-551.

GUILLORÉ P. 1980 - Méthode de fabrication mécanique et en série des lames minces. Institut National Agronomique, Paris, Département des Sols, 22 p.

HARRIS Ch. and COOK J.D. 1988 - Micromorphology and microfabrics of sorted circles, Jotunheimen, southern Norway. In «Proceeding Fourth International Conference on Permafrost», Fairbanks, Alaska, National Academy Press, Washington D.C., p. 445-449.

JOHANSSON C.E. 1963. Orientation of pebbles in running water. A laboratory study. Geografiska Annaler, XLV (2-3): 85-112.
LAVILLE H. 1973 - Climatologie et chronologie du Paléolithique en Périgord : étude sédimentologique de dépôts en grottes et sous abris. Thèse Doctorat d'Etat, $\mathrm{n}^{\circ}$ 400, Université de Bordeaux 1, 720 p. roneo.

LENOBLE A. 2005 - Ruissellement et formation des sites préhistoriques. BAR International Series 1363, $216 \mathrm{p}$.

MELLARS P. 1996 - The Neandertal legacy. An archaeological perspective from Western Europe. Princeton University Press, New Jersey, $471 \mathrm{p}$.

MERMUT A.R. and ST ARNAUD R.S. 1981 - Microband fabric in seasonally frozen soils. Soil Science Society of America Journal, 45/B : 578-586.

MOSS A.J. and WALKER P.H. 1978 - Particle transport by continental water flows in relation to erosion, deposition, soils and human activities. Sedimentary Geology, 20 : 81139.

ONAC B. P. and LAURITZEN S. E. 1996 - The climate of the last 150,000 years recorded in speleothems: preliminary results from North-Westem Romania. Theoretical and Applied Karstology, 9 : 9-21.

RAWS G. 1987 - The initiation of rills on plane beds of noncohesive sediments. Catena-Supplement $8: 107-118$.

RENAULT Ph. 1967 - Contribution à l'étude des actions mécaniques et sédimentologiques dans la spéléogénèse. Annales de Spéléologie, 22 (2) : 209-267.

ROLLAND N. 1981 - The interpretation of Middle Palaeolithic variability. Man, 16, p. 15-42.

SCHWARCZ H.P. and BLACKWELL B. $1983-{ }^{230} \mathrm{TH} /{ }^{234} \mathrm{U}$ age of a Mousterian site in France. Nature, 301 : 236-237.

SCOGING H. 1989 - Runoff generation and sediment mobilisation by water. In : D.S.G. Thomas Ed., "Arid Zone Geomorphology», p. 87-116.

TEXIER J.P. 1996 - Présence d'un réseau de grands polygones au sud de l'estuaire de la Gironde (France) : interprétation et implications paléoclimatiques. Géographie Physique et Quatemaire, 50(1) :103-108.

TEXIER J.P. 2001a - Sédimentogénèse des sites préhistoriques et représentativité des datations numériques. Actes des XXlèmes rencontres internationales d'Antibes «Datation», J.N. Barandon, P. Guibert, V. Michel eds, Editions APDCA, p. 159-175.

TEXIER J.P. 2001b - Réinterprétation géologique de quelques sites paléolithiques de référence du Périgord (France). Implications paléoclimatiques. In : XIVème congrès de l'Union Internationale des Sciences Préhistoriques et protohistoriques, Liège, 2-8 septembre 2001, Préactes, p. 74. 
TEXIER J.P. 2003a - Les sites acheuléens et moustériens de référence du Périgord : nouvelle lecture géologique et implications paléoenvironnementales. Colloque «Données récentes sur les modalités de peuplements et sur le cadre chronostratigraphique, géologique et paléogéographique des industries du Paléolithique inférieur et moyen en Europe», Rennes (22-25 septembre 2003).

TEXIER J.P. 2003b - Le contexte géologique des sites paléolithiques de plein air du Neuvicois. Mélanges Gaussen, Bulletin de la Société Préhistorique AriègePyrénées, LVIII : 133-142.

TEXIER J.P. et BERTRAN P. 1993 - Données nouvelles sur la présence d'un pergélisol en Aquitaine au cours des dernières glaciations. Permafrost and Periglacial Processes, 4(3) : 183-198.

TEXIER J.P., DELPECH F. et RIGAUD J.Ph. 1999 Programme collectif de recherche "Litho- et bio-stratigraphie de quelques sites paléolithiques de références $d u$ Périgord Rapport final, S.R.A. Aquitaine, 38 p.

TEXIER J.P., KERVAZO B., LENOBLE A., NESPOULET R. 2004 - Sédimentogenèse des sites préhistoriqus du Périgord. Association des Sédimentologue Français éd., 69 p.

VAN VLIET-LANOË B. 1976 - Traces de ségrégation de glace en lentilles associées aux sols et phénomènes périglaciaires fossiles. Biuletyn Peryglacjalny, 26 : 41-54.
VAN VLIET-LANOË B. 1985 - Frost effects in soils. In «Soils and Quaternary landscape evolution», J. Boardman ed., Wiley and Sons, p. 118-158.

VAN VLIET LANOE B. 1988 - Le rôle de la glace de ségrégation dans les formations superficielles de l'Europe de l'ouest. Editec, Caen, 2 tomes, 854 p.

VAN VLIET LANOË B., COUTARD J.P. et PISSART A. 1984 - Structures caused by repeated freezing and thawing in various loamy sediments. A comparison of active, fossil and experimental data. Earth Surface Processes and Landforms, 9 : 553-565.

VERRECCHIA E.P. and VERRECCHIA K.E. 1994 - Needlefiber calcite : a cristal review and a proposed classification. Journal of Sedimentary Research, A64(3) : 650-664.

WHALLEY W.B. 1984 - Rockfalls. In "Slope Instability», D. Brundsen and B. Prior Eds, Wiley and Sons Ltd, p. 217-256.

WIEDER M. and YAALON D.H. 1982 - Micromorphological fabrics and developmental stages of carbonate nodular forms related to soil characteristics. Geoderma, 28 : 203-220.

WHITE W.B. 1988 - Geomorphology and hydrology of karst terrains. Oxford University Press, $464 \mathrm{p}$. 
